Version of Record: https://www.sciencedirect.com/science/article/pii/S0022519318305903 Manuscript_6ad801002cfb1ef4aae6389b39408e96

\title{
Spatio-Genetic and Phenotypic Modelling Elucidates Resistance and Re-Sensitisation to Treatment in Heterogeneous Melanoma
}

\author{
Arran Hodgkinson ${ }^{\mathrm{a}, \mathrm{b}, *}$, Laurent Le $\mathrm{Cam}^{\mathrm{b}}$, Dumitru Trucu ${ }^{\mathrm{c}}$, Ovidiu Radulescu $^{\mathrm{a}}$ \\ ${ }^{a}$ Dynamique des Interaction Membranaires Normales et Pathologique, Université de \\ Montpellier, Montpellier, France \\ ${ }^{b}$ Institut de Recherche en Cancérologie de Montpellier, Montpellier, France \\ ${ }^{c}$ Division of Mathematics, University of Dundee, Dundee, Scotland
}

\begin{abstract}
Although novel targeted therapies have significantly improved the overall survival of patients with advanced melanoma, understanding and combatting drug resistance remains a major clinical challenge. Using partial differential equations, we describe the evolution of a cellular population through time, space, and phenotype dimensions, in the presence of various drug species. We then use this framework to explore models in which resistance is attained by either mutations (irreversible) or plasticity (reversible). Numerical results suggest that punctuated evolutionary assumptions are more consistent with results obtained from murine melanoma models than gradual evolution. Furthermore, in the context of an evolving tumour cell population, sequencing the treatment, for instance applying immunotherapy before BRAF inhibitors, can increase treatment effectiveness. However, drug strategies which showed success within a spatially homogeneous tumour environment were unsuccessful under heterogeneous conditions, suggesting that spatio-environmental heterogeneity may be the greatest challenge to tumour therapies. Plastic metabolic models are additionally capable of reproducing the characteristic resistant tumour volume
\end{abstract}

\footnotetext{
* Corresponding author

Email addresses: arran.hodgkinson@umontpellier.fr (Arran Hodgkinson), laurent.lecam@inserm.fr (Laurent Le Cam), trucu@maths.dundee.ac.uk (Dumitru Trucu), ovidiu.radulescu@umontpellier.fr (Ovidiu Radulescu)
}

Preprint submitted to Journal of Theoretical Biology

November 6, 2018

(C) 2018 published by Elsevier. This manuscript is made available under the CC BY NC user license https://creativecommons.org/licenses/by-nc/4.0/ 
curves and predicting re-sensitisation to secondary waves of treatment observed in patient derived xenograft (PDX) melanomas treated with MEK and BRAF inhibitors. Nevertheless, secondary relapse due to a pre-adapted subpopulation, remaining after the first wave of treatment, results in a more rapid development of resistance. Our model provides a framework through which tumour resistance can be understood and would suggest that carefully phased treatments may be able to overcome the development of long-term resistance in melanoma.

Keywords: Systems Biology, Cancer Evolution, Melanoma, Heterogeneity, Spatio-Structural Models

2018 MSC: 00-01, 99-00

\section{Introduction}

\subsection{Mathematical Background}

The vast majority of existing, quantitative models of drug resistance are based on discrete stochastic mechanisms of evolution, which fail to take into ac-

5 count the intermediary stages and continuous nature of phenotypic development $[1,2,3,4]$.

Of the continuous models, several provide insights into the dynamics of evolutionary processes but are often restricted to single cell or non-spatial population models $[5,6]$, necessarily containing space averaging assumptions (wellstirred reactor hypothesis). Of these models, few take into account the prominent theory of $\mathrm{PE}[7]$ or have the depth to explain its significance in the context of drug resistance. Herein, we present a continuous spatio-structuro-temporal model to describe both the dynamics of the population of evolving tumour cells as a whole and how targeted therapy can produce resistant strains. We further use the model to recommend future strategies for prevention of this process.

One recent study has further looked at the effect of diffusion-based drug gradients on the effective outcome of population diversity and heterogeneity [8]. This heterogeneity is evident in the biological literature but is yet to be explained by existing mathematical models. 
A new addition to the variety of available bio-mathematical modelling frameworks has been spatio-structuro-temporal modelling, introduced by Domschke et al. [9] and later subjected to higher-dimensional simulation and numerical analysis [10]. This allows one to represent not only the spatial aspects of a population but also, simultaneously, some underlying aspect of its structure, giving one more insight into the co-evolution of these characteristics. This model has since been extended further [11] but has not yet been used to look at intrinsic properties of tumours, with respect to their systematic resistance to targeted therapies.

\subsection{Biological Motivation}

Current mathematical abstractions of the biological paradigm for drug resistance characterise the biological system as existing in a series of discrete states; perhaps susceptible cells, cells with resistance to drug 1 , cells with resistance to drug 2 , and cells with resistance to both drugs. This discrete interpretation (Fig. 1a), however, is not born out in experimentation since the observation of cells

35 under the influence of any given drug will demonstrate a spectrum of response patterns. The common assumption that cells instantaneously realign themselves to a 'resistant' phenotype also appears to presuppose the eventual survival of such cells. Moreover, gene expression levels of a given cell population submitted to treatment do not appear to exhibit strong qualitative differentiation and are more accurately described as a continuum.

Therefore, we take an alternative approach to modelling wherein we consider the cellular population as a single population which is continuously variable through some structural dimension, as opposed to previous discretised descriptions (Fig. 1b). The structural dimension can be understood as a set of variables characterizing the cell state at a molecular and/or phenotypic level. Within the cell population, subgroups are differentially sensitive to drugs and may exhibit differing proliferative and migratory behaviours, more generally. This gives us extended scope to model the more nuanced aspects of the heterogeneous cellular pathways towards resistance and invasion of the surrounding tissue in cancer. 


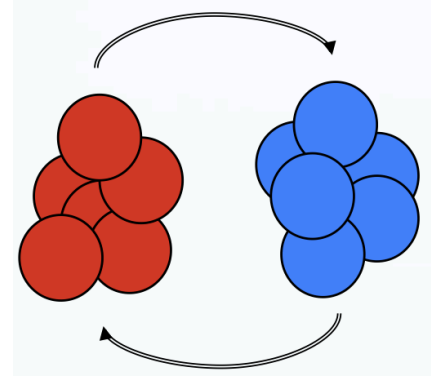

(a)

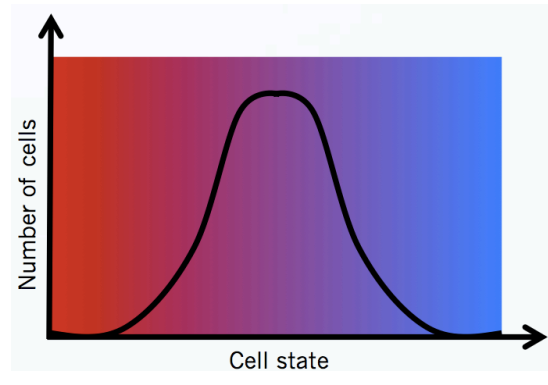

(b)

Figure 1: Diagrams indicative of (a) a discrete paradigm, wherein a cell undergoes instantaneous and complete transitions between healthy and resistant states, and (b) a continuous paradigm, in which a cell undergoes a continuous alteration between two extreme healthy and resistant states and is capable of inhabiting all points between these two extremes.

In the proceeding section (Section 2), we present a general mathematical approach to modelling biological cancer systems whose spatial and structural dynamics are coupled and introduce the various terms within this system. Moving forward we provide a possible application for this model in the study of systems who develop resistance through the sequential mutation of particular oncogenes and the effects of BRAF inhibitors and immunotherapies on this development (Section 3). The results for this mutational model are then studied in detail, with particular interest given to the effects of the order and methodology of treatment and heterogeneity in the tumour environment (Section 4). Next, we provide a second possible application for this model in the study of systems whose metabolism of certain nutrients, particularly the metabolism of glucose through glycolysis or oxidative phosphorylation, shapes their response to drugs, resulting in a plastically resistant system (Section 5). We then explore results coming from this metabolically plastic system with a specific view to understanding the effect of treatment of spatial and metabolic heterogeneity and the resulting responses to treatment (Section 6). Finally we discuss the results from both of these systems in the wider contrast and the ramifications of this current study (Section 7). 


\section{Presentation of the General Model}

Herein, we present a mathematical model that contains

70 
Mathematically, we employ a multi-dimensional framework which allows for the coupling of spatial dynamics, in $x$, with other biological or biochemical dynamics in the cells themselves, which we call structural dynamics and denote by $y$. Then we can use an existing mathematical framework $[9,11]$ to deduce that the change in cell density $c(t, x, y)$ is given by the continuity equation

$$
\frac{\partial}{\partial t} c(t, x, y)=\nabla_{x} \cdot \underbrace{F(c, v, \bar{m})}_{\text {Spatial Flux }}+\nabla_{y} \cdot \underbrace{G(y, c, v, \bar{m}, \bar{p})}_{\text {Structural Flux }}+\underbrace{S(y, c, v, \bar{m}, \bar{p})}_{\text {Source }},
$$
through attraction to biochemicals or substrate components, respectively. The diffusion, chemotactic, and haptotactic rate constants are then given by $D_{c}, \bar{\chi}_{m}$, 
and $\chi_{v}$, respectively. This may be mathematically represented as the following term:

$$
F(c, v, \bar{m})=D_{c} \nabla_{x} c+c(1-\rho(c, v))\left(\nabla_{x}\left(\bar{\chi}_{m} \cdot \bar{m}\right)+\nabla_{x} \chi_{v} v\right) .
$$

As in [13], the chemo- and haptotactic fluxes are volume constrained and vanish when the collective spatial volume reaches a maximum capacity that, without loss of generality, is considered equal to one. A simple way to take this constraint into account is to consider that these two fluxes are proportional to $1-\rho$, where $\rho(c, v)$ is defined as in (2).

\subsection{Structural flux of the cellular population}

The structural flux is the sum of two terms, an advection flux and a structural diffusion flux, corresponding to biased and unbiased evolution in the structure space, respectively.

In order to define the advection flux we introduce the function $\Psi(y, \bar{m}, \bar{p})$, representing the normalized structural velocity, who is dependent upon the population's structural distribution, the local nutrient concentration and the local concentration of drugs. Given some maximal rate for the population's velocity through the structural dimension, $r_{\mu}$, the structural velocity shall be given by $r_{\mu} \Psi(y, \bar{m}, \bar{p})$, where the normalized structural velocity satisfies $|\Psi(y, \bar{m}, \bar{p})| \leq 1$. The structural advection flux term is the product of the structural velocity and the cell distribution density and reads

$$
G_{a}(y, c, v, \bar{m}, \bar{p})=r_{\mu} \Psi(y, \bar{m}, \bar{p}) c .
$$

In this paper, we shall consider one cellular system in which behaviour is adapted through the accumulation of consecutive mutation (Section 3) and one in which a cell may plastically evolve its behavioural phenotype through metabolic reprogramming dynamics (Section 5). For each of these scenarios, it will be necessary to define a distinct and biologically relevant form for the function $\Psi(y, \bar{m}, \bar{p})$. 
Diffusion in structural space can occur as the result of a stress, following a change of environmental conditions. In order to adapt to the environment, the population tends to diversify its behaviour which leads to an increase in spread of the $y$-space cell distribution. This diversification of behavior can be phenomenologically described by a structural diffusion matrix $\Sigma(y, \bar{m}, \bar{p})$. The structural diffusion flux is supposed to satisfy Fick's law and reads

$$
G_{d}(y, c, v, \bar{m}, \bar{p})=-\Sigma(y, \bar{m}, \bar{p}) \nabla_{y} c .
$$

Although structural diffusion is possible both in a mutational and a metabolic context, in this paper we will consider it only in relation to metabolic remodeling.

The total structural flux is the sum of the structural advection and structural

155 diffusion terms and reads

$$
G(y, c, v, \bar{m}, \bar{p})=G_{a}(y, c, v, \bar{m}, \bar{p})+G_{d}(y, c, v, \bar{m}, \bar{p}) .
$$

\subsection{Source/Sink terms for the cellular population}

The growth of any given cell will be dependent on an assortment of intracellular and environmental factors, including its structural state, $y$; the availability of nutrients, $\bar{m}$; and the volume surrounding the cell which has not yet been filled, $\rho(c, v)$. Therefore, we write the growth rate of the population generically as $\Phi(y, \bar{m}, c, v)$ such that we may define its particular dynamics for the considered scenario. It is important to remember that this term accounts only for growth of the cell population and not the negative growth caused by the introduction of drugs.

It is clear that, since drugs are typically designed to exploit a particular behaviour or dependence of a given cancerous population, its effectiveness will be dependent upon the current structural state of the cell, $y$. We account for 170 the effect of drugs on the cellular population, then, by taking the product of the cellular apoptosis rate, the drugs' effectiveness functions, and the respective 
local drug concentrations $\delta_{c} \bar{p}(t, x) \bar{f}(y)$. Multiplying this by the cellular concentration, itself, will yield the degradative sink. As such, the entire source/sink term may be written mathematically as

$$
S(y, c, v, \bar{m}, \bar{p})=\Phi_{c}(y, \bar{m}, c, v) c-\delta_{c} \bar{f}(y) \cdot \bar{p} c .
$$

175

Since, in this particular study, we are interested in the effects of structural heterogeneity on the success of a given cancer population the normalized structural velocity, $\Psi(y, \bar{m}, \bar{p})$; structurally-dependent growth function, $\Phi_{c}(y, \bar{m}, c, v)$; and the structurally-dependent drug effectiveness function, $\overline{\mathfrak{f}}(y)$, are of most indoes not degrade the ECNE, the degradation constant $\delta_{v, m_{i}}=0$. Our PDE for the ECNE dynamics is then given by

$$
\frac{\partial v}{\partial t}=\Phi_{v}(c, v) v-\bar{\delta}_{v} \cdot \bar{m} v .
$$

Spatial dynamics of the molecular species vector, $\bar{m}(t, x)$, are given simply by diffusion with its rate vector $\bar{D}_{m}$. Chemical species are then produced either by the ECNE, and connected network of capillaries, $v(t, x)$, or the cellular species, $c(t, x, y)$, with rates dependent on $y$ such that its general expression may be given by the function $\bar{\Phi}_{m}(y, \bar{m}, c, v)$. We then assume that environmental factors, 
which are not directly accounted for, shall contribute to the degradation of molecular species with respective degradation rates of $\bar{\delta}_{m}$. Dynamics for the molecular species are then collectively written as

$$
\frac{\partial \bar{m}}{\partial t}=\nabla_{x} \cdot \operatorname{diag}\left(\bar{D}_{m}\right) \nabla_{x} \bar{m}+\int_{\mathcal{P}} \bar{\Phi}_{m}(y, \bar{m}, c, v) d y-\operatorname{diag}\left(\bar{\delta}_{m}\right) \bar{m} .
$$

Finally, spatial dynamics for the drug species vector, $\bar{p}(t, x)$, are also given by diffusive dynamics, with a rate vector $\bar{D}_{p}$. We then represent the input of drug species to the population as a vectorial function, $\bar{\theta}(t, x)$, which is to define the drug regimen used by the clinician/scientist in treating the tumour. This will normally be given by a sum of Dirac delta functions centred at the time of injection of the drug but may be given by other forms and will be particular to the experiment that the model attempts to replicate. Finally, we assume that the drug's effect on the cellular system requires the drug to be taken in by cells and systematically degraded during apoptosis. Therefore, given a drug degradation vector, $\bar{\delta}_{p}$, this degradation shall be committed by the nonstructured cellular population, written as the integral $\int_{\mathcal{P}} c d y$. The complete equation for drug dynamics is then given by

$$
\frac{\partial \bar{p}}{\partial t}=\nabla_{x} \cdot \operatorname{diag}\left(\bar{D}_{p}\right) \nabla_{x} \bar{p}+\bar{\theta}(t, x)-\operatorname{diag}\left(\bar{\delta}_{p}\right) \bar{p} \int_{\mathcal{P}} c d y .
$$

\subsection{Summary of the General Mathematical Model}


We then write the system of PDEs as

$$
\left\{\begin{aligned}
\frac{\partial c}{\partial t} & =\underbrace{\nabla_{x} \cdot\left[D_{c} \nabla_{x} c+c(1-\rho(c, v))\left(\nabla_{x}\left(\bar{\chi}_{m} \cdot \bar{m}\right)+\nabla_{x} \chi_{v} v\right)\right]}_{\text {Spatial Flux }} \\
& +\underbrace{\nabla_{y} \cdot \Sigma(y, \bar{m}, \bar{p}) \nabla_{y} c-r_{\mu} \nabla_{y} \cdot \Psi(y, \bar{m}, \bar{p}) c}_{\text {Structural Flux }}+\underbrace{\Phi_{c}(y, \bar{m}, c, v) c}_{\text {Growth }}-\underbrace{\delta_{c} \overline{\mathcal{f}}(y) \cdot \bar{p} c}_{\text {Drug Influence }} \\
\frac{\partial v}{\partial t}= & \underbrace{\Phi_{v}(c, v) v}_{\text {ECNE Remodelling }}-\underbrace{\bar{\delta}_{x} \cdot \bar{m} v}_{\text {MMP Degradation }} \\
\frac{\partial \bar{m}}{\partial t}= & \underbrace{\nabla_{x} \cdot \operatorname{diag}\left(\bar{D}_{m}\right) \nabla_{x} \bar{m}}_{\text {Spatial Diffusion }}+\underbrace{\int_{\mathcal{P}}^{\int_{\Phi_{m}}(y, \bar{m}, c, v) d y}-\underbrace{\operatorname{diag}\left(\bar{\delta}_{m}\right) \bar{m}}_{\text {Natural Degradation }}}_{\text {Chemical Synthesis }} \\
\frac{\partial \bar{p}}{\partial t}= & \underbrace{\nabla_{x} \cdot \operatorname{diag}\left(\bar{D}_{p}\right) \nabla_{x} \bar{p}}_{\text {Spatial Diffusion }}+\underbrace{\bar{\theta}(t, x)}_{\text {Drug Input }}-\underbrace{\operatorname{diag}\left(\bar{\delta}_{p}\right) \bar{p} \int_{\mathcal{P}} c d y}_{\text {Drug Degradation }} .
\end{aligned}\right.
$$

This system of equations (11) is considered together with no-flux boundary

conditions in $c, m$, and $\bar{p}$. In the case of $c$ we consider zero spatial fluxes, and zero structural fluxes on the boundaries of the spatial and structural domains, respectively.

In the following sections, we present an intuitive explanation for the origin of the equations and relations used for two particularisations of this general mathematical system, along with a thorough description of the biological evidence for such phenomena.

\section{Mutational Evolution and the Establishment of Drug Resistance}

Melanoma is one of the most common cancers of the skin and approximately $50 \%$ of melanomas contain a mutation in an oncogene known as BRAF, often appearing at codon 600 [14]. Therefore, BRAF inhibitors (BRAFi) have been the major drug of choice in treating advanced melanoma tumours and their 
had mixed results due to the frequent presence of BRAFi resistant phenotypes existing as subspecies within the overall melanoma species $[15,16,17,18,19]$. The resistance mechanism could involve activation of collateral signaling pathways when the main signaling is inhibited [20]. For this reason, simultaneous resistance [20].

Moreover, recent studies suggest that intravenously injected, water-soluble MAPK activator can overcome, to some extent, the resistance to BRAFi [21]. This, in turn, suggests that the penetration to the inner domain of the tumour over, BRAFi is often used in combination with MEKi in order to target several mechanisms of activation within the MAPK pathway.

In animal models, as well as in patients, relapse occurs systematically several months after treatment with BRAFi [22]. Studies have shown that the $[23,24]$, which may suggest that cancer cells have acquired a resistant state before application of BRAFi.

The order in which drugs are supplied to the tumour may also have a significant effect on the clinical outcome. Progression-free survival rates were higher among those receiving immunotherapy prior to BRAFi than vice versa [25] whereas one particular study looking at treatment with immunotherapy and BRAFi found that preceding BRAFi with immunotherapy does not alter the effectiveness of the drug. Treatment with immunotherapy post-BRAFi, however, gives the patient a particularly poor clinical outcome [26].

One strategy for drug application on the premature tumour has been shown to apparently forestall the resistance to BRAFi. This methodology involved applying the drug to the tumour, for a period of time appearing to demonstrate a reduction in the tumour volume, before removing the drug and repeating the process, again. This method showed mixed results although a significant 265

number of the resistant tumours did not survive the treatment [27]. 
Tumours that have been shown to have innate BRAFi resistance have further been shown to have increased incidence of mutations in genes known as NRAS $[28,29]$ and PTEN [30], respectively.

In human liver cells, those cells with an induced PTEN knockdown have been shown to increase the rates of Akt phosphorylation and, importantly, to inhibit Foxo1 signalling [31]. Foxo1, in return, is a transcription factor responsible for mediating the T-cell response to healthy cells [32]. In CD8 ${ }^{+}$T-cells, Foxo1 has been shown to have an intrinsic role in establishing long-lived memory programs that are essential for developing cells capable of immune reactivation during secondary responses to infection [33, 34].

On the other hand, the gene encoding for phosphatidylinositol 3-kinase (PI3K), whose oncogenic pathway is inhibited by PTEN expression, has been shown to reduce the cytokine expression in cells [35], thereby reducing the inflammatory response of the surrounding tissue and limiting T-cell recruitment to the site. Cells with a PTEN deletion might then be protected from immune response through mediation of cytokines and the local apoptosis induced through PI3K/Akt signalling, which may subsequently be overcome by the induction of T-cell hyperactivity induced by ipilimumab - a melanoma-specific immune enhancer therapy.

285 Therefore, it is possible that melanoma cells undergo sequential genetic alterations in BRAF and PTEN, respectively, and that the pattern in which these mutations occur, along with considerations with respect to competition for nutrients, could explain the build up of resistance to the combined effects of BRAFi and ipilimumab anti-oncogenic treatments.

290 It may also be that BRAF mutated cells, as a result of causal genomic instability, acquire NRAS mutations which confer resistance. This change, for example, was observed within ovarian cell lines and was predicted to have formed as a result of exon 11 BRAF mutations being insufficient to satisfactorily activate the MAPK pathway, requiring additional NRAS activity [36]. Furthermore, BRAF V600E cells have sufficient MAPK activity such that they do not necessitate supplementary mutation and, as such, display a more positive response 
to therapy [37], which is supported in the majority of cases of melanoma with a native BRAF mutation [38]. Yet, despite the fact that BRAF and NRAS mutations are described commonly as "mutually exclusive", NRAS mutations appear in increased numbers of BRAFi resistant tumours [29].

In our model, we interpret the primary and consequent mutation to be that in BRAF and assume, further, that the cell will acquire some further mutation capable of conferring resistance to BRAF inhibitors.

\subsection{Interpreting the structural dimension for a mutational system}

In order to understand how this system of sequential mutations contributes to the cancer cell population's success at avoiding targeted and immune-enhancement therapies, we must first interpret the structural-, $y$-, dimension. So, letting the cellular population be given by a function $c(t, x, y)$ and the ECNE concentrations be given by the function $v(t, x)$, with $\bar{m}(t, x)$ and $\bar{p}(t, x)$ giving the molecular and drug species, respectively, we observe the bio-mathematical dynamics of such a system in the structure space, $\mathcal{P}$.

We also assume, that the cellular species will migrate unidirectionally through the structure space, which is to say that mutations are irreversible. Let the structural mutation variable and space, then, be given by the interval $y \in \mathcal{P}=$ $[0,1]$, such that $y=0$ and $y=1$ give the extreme states of primary tumour (or as yet without a mutation) and resistant, respectively. For ease, let us also define that $y=1 / 2$ defines a BRAF mutation and the state at which the cellular species is most sensitive to BRAFi. Realistically, the ipilimumab 320 immune-enhancer drug will be effective across the entire spectrum of mutations but we assume it to be most effective posterior to BRAF mutation and prior to complete consolidation of resistant features at $y=1$.

\subsection{Growth, ECNE remodeling and drug dosing in a mutational system}

Let $\rho(t, x)$ be defined as in 2 such that the growth of the cellular species, $c(t, x, y)$, shall be dependent upon the unoccupied local volume, $1-\rho(t, x)$ and 
is also dependent upon the nutritional species, $m_{2}(t, x)$, being above a given threshold, $\theta_{m_{2}}$. The cellular growth rate, with an overall rate parameter $\phi_{c}$, is then written as

$$
\Phi_{c}(y, \bar{m}, c, v)=\phi_{c}\left(m_{2}-\theta_{m_{2}}\right)(1-\rho(c, v)),
$$

where we consider that growth, in this case, is not dependent upon the mutational status of the cells $y$.

Again, the ECNE remodelling takes place within the unoccupied portion of the local available volume, $1-\rho(t, x)$, and with a rate constant $\phi_{v}$, such that

$$
\Phi_{v}(c, v)=\phi_{v}(1-\rho(c, v)) .
$$

Although we assume here that ECNE remodelling is only dependent on the unoccupied volume, we recognise that more realistically this could depend on fibroblast cells and ultimately on the cell phenotype represented by $y$. Therefore, future iterations of this modelling approach could incorporate more complex remodelling through a redefinition of the $\Phi_{v}$ term.

We then endow the system with two molecular species. $m_{1}$ is a species that ${ }^{40}$ is secreted by the cell species and will act to degrade the ECNE. This can be thought of as a matrix metalloproteinase (MMP) which acts to break-down the ECNE. $m_{2}$ is a species which is secreted by the ECNE and acts to the benefit of the cellular species. This chemical species can be thought of as a nutrient or growth factor, the presence of which aids the growth of cellular species.

345 We further assume that more mutated and aggressive cellular populations will produce MMP molecules at a greater rate, such that their production is proportional to $y$, and that the overall rate constant is given by $\phi_{m_{1}}$. We write this as

$$
\Phi_{m_{1}}(y, \bar{m}, c, v)=\phi_{m_{1}}\left(1-m_{1}\right) y c .
$$

Nutrient, or nutritional species, are produced by the ECNE and with a rate of $\phi_{m_{2}}$, such that

$$
\Phi_{m_{2}}(y, \bar{m}, c, v)=\phi_{m_{2}}\left(1-m_{2}\right) v .
$$


We assume an instantaneous introduction of drug species through the vasculature, which we assume to be proportional to ECNE concentration. The instantaneous nature of this drug introduction mean that we may write this as a Dirac delta function $\breve{\delta}(t-\tau)$ centered at some time $\tau$, whilst its introduction through 355 the vasculature of the ECNE is represented by proportionality to $v(t, x)$. Then, given that the number of doses of some $j^{\text {th }}$ drug species, $p_{j}(t, x)$, is a natural number, $N_{p_{j}} \in \mathbb{N}$, we write that the doses are given at the ordered set of time points $\left\{\tau_{j, 1}, \tau_{j, 2}, \ldots, \tau_{j, N_{p_{j}}}\right\}, \tau_{j, 1}<\tau_{j, 2}<\cdots<\tau_{j, N_{p_{j}}}$. Then the mathematical expression for drug dosing is given by

$$
\theta_{j}(t)=v(t, x) \sum_{k=1}^{N_{p_{j}}} \breve{\delta}\left(t-\tau_{j, k}\right) .
$$

360

\subsection{Mutational dynamics in melanoma: Phyletic gradualism or punctuated equilibria?}

Patterns in genetic evolution can generally be categorised by the theory of the theory of Darwinian evolution by natural selection and seeks to explain the variety of species by continuous gradual change [39, 40]. PE, on the other hand, is a currently prominent theory in evolutionary biology that seeks to explain the nature of evolution by natural selection through the prism of large plain cancer progression. These theories added chromosome instabilities and selection processes to the older idea that cancer results from an accumulation of somatic mutations [46]. Furthermore, the gradual accumulation of mutations over time has been challenged by recent evidence that tumours evolve by 
lesions[48, 49]. These findings suggest that cancer genomes evolve by PE, being thus able to acquire quickly new capacities such as invasiveness and drug resistance $[50,51,52]$. This $\mathrm{PE}$ can be explained on a more microscopic level by assuming that the intermediary stages of mutation, although significant, happen ingly, gradualism would convey a sense of regular and linear progression within the phyletic tree of the cancer species with little or no change in the rate of mutation.

Single-cell genetic analysis reveals clonal frequencies and phylogeny patterns of evolving tumours $[53,54,55,56]$. Various clones have heterogeneous survival properties in the presence of drugs; as a result of this selection pressure, drug resistant clones can become predominant. For instance, mutations of the genes BRAF and NRAS are well known to be driver mutations for melanoma [57, $58,59,60]$. The wealth of literature on melanomal branching evolution has identified BRAF as the major trunk driver mutation and NRAS or MEK1 as the major branch driver mutations $[61,62]$. It has also been recognised that the targeted treatment of genetically evolved melanoma results in a reduction of their heterogeneity [59], as only drug resistant genetic variants survive, but not in their eradication.

For the sake of simplicity, in our model we consider that only two mutations can occur, and that their occurrence is sequential.

\subsection{A structural flux function in a mutational system}

To clarify the mathematical evolution of our cancer cell population, we must more clearly define how the population changes in structure, through the normalized structural velocity $\Psi(y, \bar{m}, \bar{p})$ (further discussion in Appendix A.1). This function is intended to represent the velocity of any given cell in the $y$-direction (in other words, the mutation rate), for given current structural state (y-coordinate) and local nutritional condition, $m_{2}(t, x)$. We shall define a 


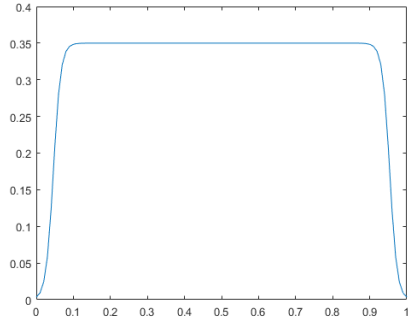

(a)

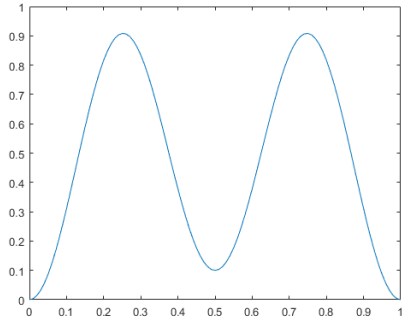

(b)

Figure 2: Normalized structural velocity, $\Psi(y, \bar{m}, \bar{p})$ for the (a) phyletic gradualism (PG) and (b) punctuated equilibrium (PE) assumptions.

In the case of $\mathrm{PG}$, we wish for the evolution of this population to be steady and regular throughout the domain, such that the mutation rate must fundamentally be constant throughout the domain. Then, in order to ensure that our population does not migrate beyond the boundaries of the domain, $y=0$ or $y=1$, we set the values of the normalized structural velocity to 0 at these locations, yielding no mutation at these biological positions (Fig. 2a).

In the $\mathrm{PE}$ case, we require for the mutation rate to be significantly greater in periods between mutational realisation that at those positions themselves. Therefore, we represent the normalized structural velocity as a bimodal function with velocity maxima positioned between the mutational states. Likewise with the PG function, however, we require for the PE paradigm to yield a 0 , non-mutational behaviour at the boundaries of the domain (Fig. 2b). Remember, given that these function represent the rate of mutation, a higher value of $\Psi(y, \bar{m}, \bar{p})$ will convey a faster rate of mutation whilst a lower value will convey a more quiescent state, where change is somewhat slower.

For the sake of simplicity, we do not consider genetic diversification and structural diffusion in this context.

\subsection{Drug effectiveness functions in a mutational system}

The drug effectiveness is given by a vector valued function $\overline{\mathfrak{f}}(y):=\left[\mathfrak{f}_{1}(y), \mathfrak{f}_{2}(y)\right]^{T}$, 


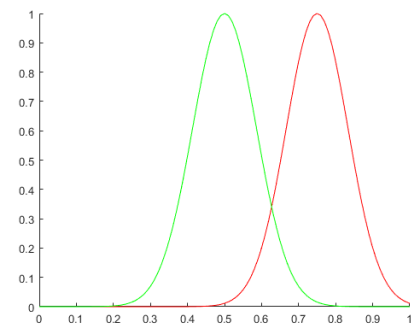

Figure 3: Distribution for the drug effectiveness functions $\mathfrak{f}_{1}(y)$ (green) and $\mathfrak{f}_{2}(y)($ red $)$.

where $\mathfrak{f}_{i}(y)$ gives the effectiveness of its corresponding $i^{\text {th }}$ drug, $p_{i}(t, x)$. For simplicity, we assume that each of these functions is given by a Gaussian function centred at its point of greatest structural significance, or the structural location in $y$ at which it is most effective against cancer cells.

Now, since $p_{1}(t, x)$ is define to be a BRAFi therapy and we have defined that the BRAF mutation is fully realised at the structural location $y=1 / 2$, we assume that $\mathfrak{f}_{1}(y)$ attains its maximal value at $y=1 / 2$ (Fig. 3 green). The considerations for ipilimumab are somewhat more numerous and difficult to entirely confirm but are, for our purposes, limited to the following. Firstly, we assume that immune cells should largely ignore healthy cells without a mutation such that there effectiveness at $y=0$ should be negligible. Moreover, we know that cancer cells will eventually become resistant even to this immune-enhancer therapy and, as such, the value of effectiveness function must be sufficiently low in the neighbourhood of $y=1$, so as to allow this resistance phenomenon to manifest. Likewise, immune cells require the expression of some protein on the surface of any given cell in order to identify its genetic properties; as such, we assume that only as the BRAF mutation becomes realised, near $y=1 / 2$, shall the ipilimumab therapy begin to have a significant effect. Given these considerations, we place the maximum of $\mathfrak{f}_{2}$ at $y=3 / 4$ (Fig. 3 red).

\section{Results for the Mutational System}

Primarily, in the application of this system to studying the death and regrowth models of tumour resistance in mice, we wished to know whether or not 
our in silico model was able to recapitulate in vivo results. In the process of exploring this potential in the model, we attempt to asses the ability of either phyletic gradualistic or punctuated equilibrium assumptions, on the tumour's evolution, were more able to consistently capture this phenomenon (Section 4.1). Secondly, we wished to test whether, given knowledge of sequenced treatments' ability to succeed in the ablation of the tumour, we could draw conclusions about the sequencing of treatments and their relative success (Section 4.2). In line with this, we tested periodic treatments to understand what the heterogeneity in initial conditions of the tumour could teach us about the outcomes for treatments (Section 4.3) and, finally, what effect a heterogeneous environment would have on these above conclusions (Section 4.4); whether results would be conserved or altered in the presence of a heterogeneous spatial conditions.

In order to test these scenarios, the in silico experimental approach was primarily as so: We began by choosing a melanoma mouse model for which one could attempt to tune our parameters and, effectively, challenge the model. The model that we chose for this task was that of Perna et al. who explored the explosive regrowth of tumours after some post-treatment dormancy period [22], amongst other things. Once we had used this in vivo model to tune and test our mathematical in silico model, we would use other biological models in order to challenge the mathematical model with no further doctoring of the mathematical model or its parameters. For this challenge we chose, initially, that of Thakur et al. [27].

Thus, we obtain that these mutations occur at maximal probabilistic rates of approximately $1.9 \times 10^{-2}$ genetic events per day. This corresponds to acquiring a genetic mutation every 40-50 days posterior to some precursor event, where we consider only 2 such events. This is supported by the fact that tumours planted in the mouse species show significant change in expression pattern after 25-45 480 days $[63,64]$, where below 40 days BRAFi was a largely successful treatment [65], and mouse models show significant behavioural change in the cancer cell dynamics after 100 days since inocculation [22].

Proliferative and degradative parameters were chosen to be in line with pre- 

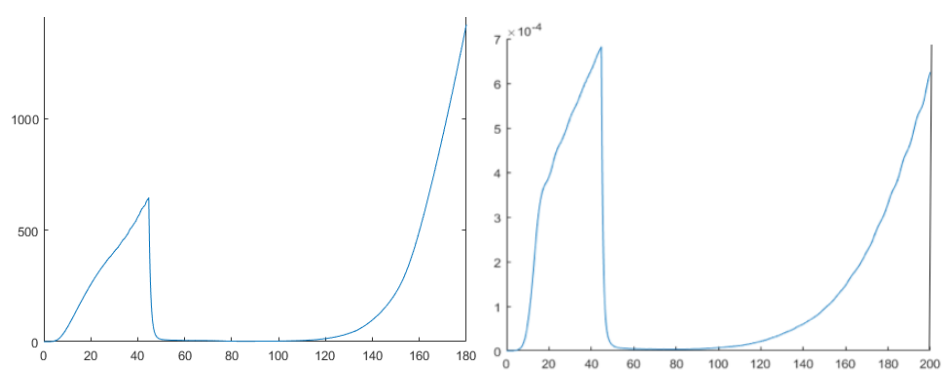

$\eta=0.05$
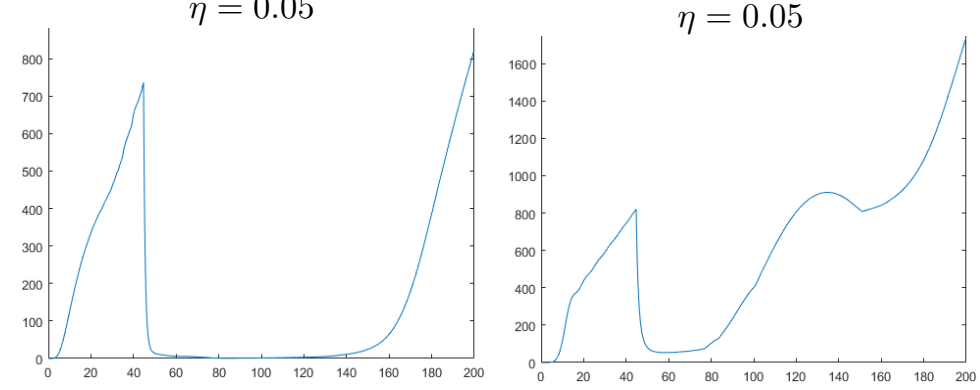

$\eta=0.05+r$

$\eta=0.05+r$

(a) Punctuated Equilibrium (PE)

(b) Phyletic Gradualism (PG)

Figure 4: Punctuated evolution is more consistent with biological results than gradual evolution. Tumour volume graphs for (a) punctuated equilibrium (PE) and (b) phyletic gradualism (PG) assumptions under a simply BRAFi therapy option applied at $t=40$ for initial conditions of $\eta=0.05$ or $\eta=0.05+r$, where $\eta$ represents the initial mean location of the tumour cells along the phenotypic dimension, and $r=0.1$ represents a perturbation.

vious models and were fine-tuned for the mouse model considered, based on tumour growth rates observed in tuning experiments [22]. All of these values are summarised in Table B.1.

\subsection{Punctuated equilibrium (PE) assumptions are more consistent with in vivo experimental results than phyletic gradualism ( $P G)$ assumptions}

Given certain initial conditions for the cellular population, namely an initial structural distribution centred at $\eta=1 / 20$, both PE and PG assumptions can give rise to the characteristic death and regrowth curves, albeit with differing characteristics (Fig. 4). In both cases, one observes an initial growth phase 
which is quickly stunted and violently reversed by the introduction of the drug species at $t=45$. This is followed by a period of dormancy or 'tolerance' before the characteristic resistant growth (or regrowth) phase, which is of particular interest to our current study. Observe, initially that the regrowth phase manifests a an earlier time point and with a faster growth rate under PE assumptions than with PG assumptions.

500

Now, observe that inducing a significant (200\%) perturbation in only the position of the initial conditions, we evoke dramatically differing behaviours from our two in silico tumours (Fig. 4). For the case of PE, the rate at which our tumour regrows to its pre-treatment volume is much slower but the death and prolonged dormancy phases are conserved between these two experiments 505 (Fig. 4a). Under the assumptions of PG, however, one observes at all time points a tumour volume with a significant positive minimum value (Fig. 4b). This shift in the volumes of tolerant tumours to be visible for all time points is not consistent with the results of comparative in vivo experiments [22] and, thusly, the initial conditions of a PG model would have to be strictly constrained to some smaller subset of possible conditions in order to maintain its relevance.

In biological, and especially in the case of in vivo, experimentation, however, the initial conditions of a given tumour or its new environment may never be strictly limited. This would suggest, due to its robustness to fluctuations in initial conditions, that the PE modelling assumption is most consistent with the

515 results of murine experimentation, since the characteristic death and regrowth curve is conserved.

\subsection{Sequencing and order of treatments are vital to their success}

In order to test the importance of the order of drug treatments on the resistance phenomenon we have first used homogeneous initial conditions for the ECNE. These conditions also preserves the spherical symmetry of the tumour when drugs are applied uniformly on the periphery. Heterogeneous initial conditions leading to non-spherically symmetric tumours will be tested in Section 4.4. 


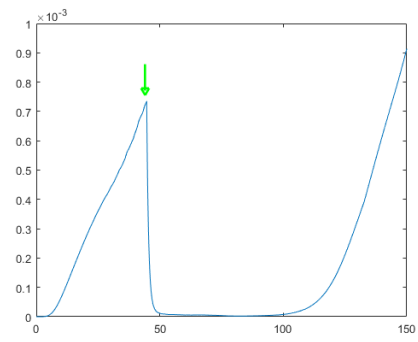

(a)

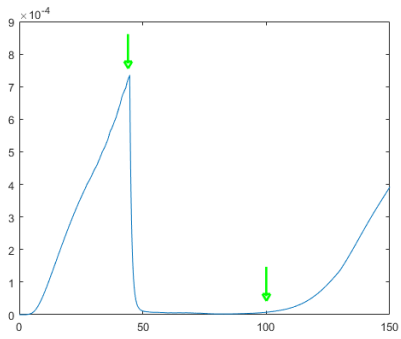

(c)

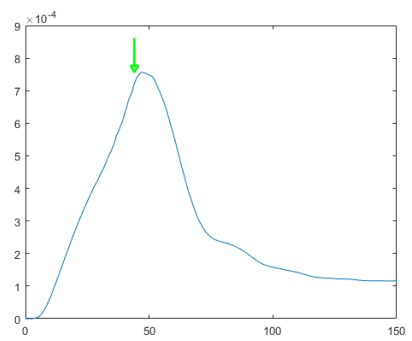

(b)

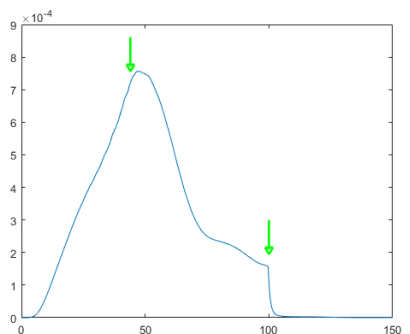

(d)

Figure 5: The sequencing of treatments is crucial to success. Overall tumour volume over time, calculated using (B.5), with the drug strategies (a) BRAFi, (b) ipilimumab, (c) BRAFi followed by ipilimumab, and (d) ipilimumab followed by BRAFi; where the drugs are applied constantly after some $t=45\left(1^{\text {st }}\right.$ green arrow) and then $t=100\left(2^{\text {nd }}\right.$ green arrow) when applicable

With that understood, in all cases and treatment scenarios the tumours initially respond to treatment, exhibiting a significant period of apoptotic degradation (Fig. 5). Experiments wherein only one treatment was used (Fig. 5a $\& 5$ b) show dramatically differing clinical treatment profiles. BRAFi treatment shows an extremely promising tumour response with almost complete erradication occuring within days of treatment but followed by an exaggerated regrowth (Fig. 5a), as seen in murine experiments. Ipilimumab therapy does not show as successful an eradication pattern at earlier time points but is more consistent in quelling its resistance and resulting regrowth (Fig. 5b), although ultimately unsuccessful in eradicating the tumour.

Observing the therapeutic strategy of utilising a BRAFi treatment followed 

5d). Although the ipilimumab post-treatment is slowing the growth of the now aggressive tumour, it may already be resistant to immunological therapies. The ipilimumab treatment followed by BRAFi post-treatment, however, appears to be extremely effective (Fig. 5d), with a negative growth rate for the tumour volume maintained as of $t=1000$ (Results not shown). This counterintuitive result may be explained as follows: Firstly, BRAFi appears extremely effective at depleting the tumour volume but is incapable of preventing the resistant escape of subpopulations to higher values of $y$ (Fig. 5a). On the other hand, ipilimumab's effectiveness function is centred at a greater value of $y$ than BRAFi's, making surviving tumour cells at lower values of $y$, where BRAFi remains effective. Therefore, these results would suggest that BRAFi should be used to destroy the tumour once its tendency towards resistance has been stemmed through ipilimumab's immunological mechanisms.

550

4.3. Oscillatory tumour volumes as a result of periodic treatments do not necessarily imply re-sensitisation

Our second experimental approach was to attempt the experiment of Thakur et al. [27] who implemented a periodic treatment regimen for their in vivo tumours. This periodic treatments managed to eradicate the death and rapid regrowth phases of those previous experiments and instead resulted in oscillatory dynamics in the tumour volume. Across several cycles of these treatments, some tumours managed to outgrow the drugs and became resistant, although more slowly, whilst others appeared to reduce their volume even over far longer timeperiods. The research team explained this by suggesting that the application of less severe treatment regimes may delay the resistance to treatment in solid tumours by failing to encourage the development of such resistance.

Likewise, in our experiments we observed an oscillatory dynamics resulting from the periodic application of smaller dosages to the tumour and subsequent 565 

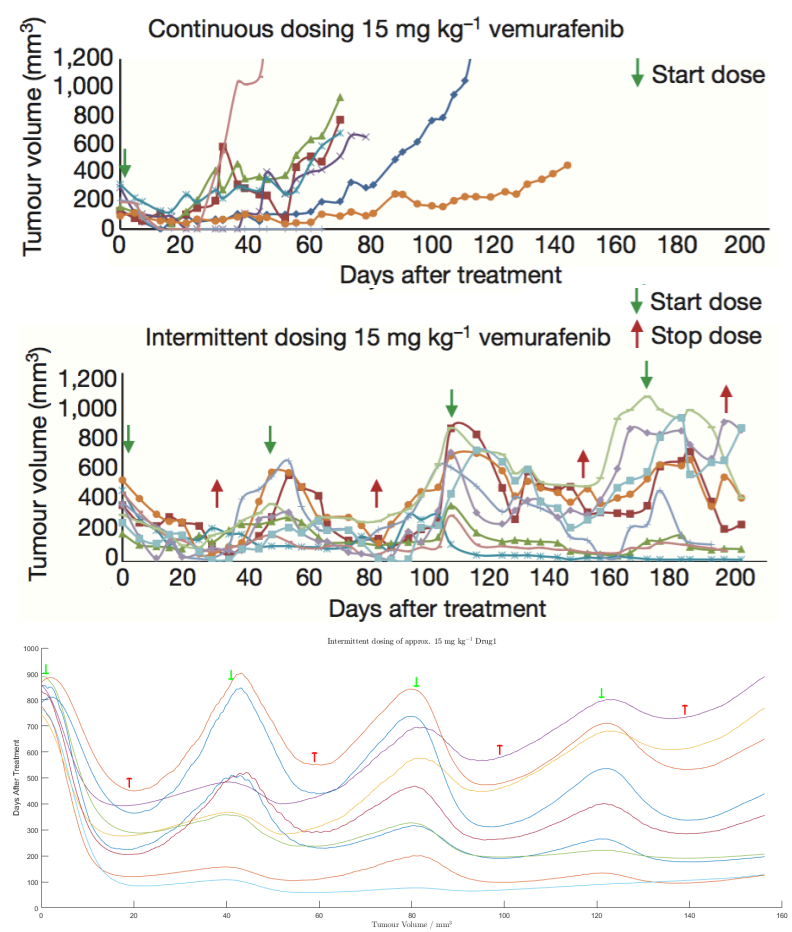

Figure 6: Oscillatory tumour volumes can emerge in the absence of re-sensitisation. The top and middle panels are figures from Das Thakur et al. [27] for in vivo melanoma tumours under an intermittent dosing strategy and the bottom panel gives the in silico results of the same experiments run using the mutational mathematical model. (Licenses applied for from Nature Publishing Ltd.)

starting $y$ positions in the initial conditions for our cancer cell population, our results gave a greater qualitative agreement with those of Thakur et al. [27]. Moreover, we found that there was a strong correlation between the average $y$-position of the initial condition and the final tumour volume at $t=160$.

These results allowed us to reinterpret this oscillatory behaviour. In our in silico model, the acquisition of resistance is certainly not delayed because cells are progressing irreversibly in the $y$ direction. In fact, what may be occurring is that in a situation where some number of cells are resistant whilst other are not, these two heterogeneous subpopulations will have to compete for available nutrients in the environment. Not only this but, together, they will consume 


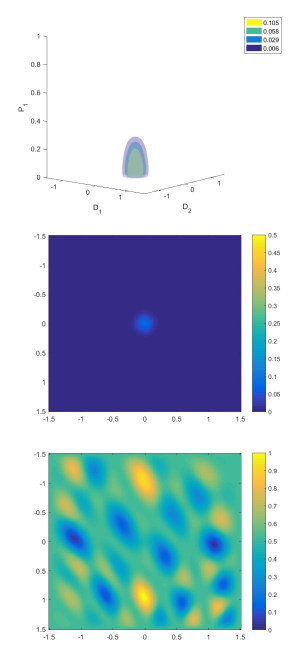

(a) $\mathrm{t}=5$
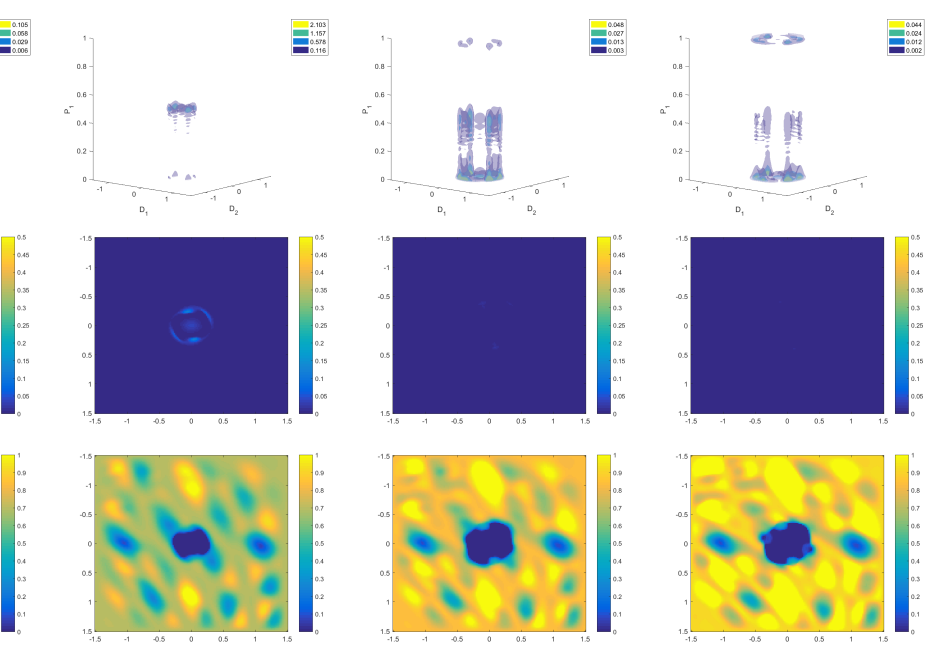

(b) $\mathrm{t}=40$

(c) $\mathrm{t}=80$

(d) $\mathrm{t}=120$

Figure 7: Spatial heterogeneity eradicates treatment success. Panels displaying (top) the structured cellular population with space across the lower plane and mutational state given along the vertical axis; (middle) the spatial cellular distribution; and (bottom) the ECNE density, where ipilimumab treatment is given at $t=40$ and BRAFi treatment is given at $t=100$, for time points $t \in\{5,40,80,120\}$ are shown.

more nutrients, leaving fewer such nutrients for the resistant subpopulation and leaving a greater subpopulation sensitive to existing treatment options. A dynamical state will be reached where the two sub-populations are oscillating while keeping their volumes bounded.

580

\subsection{Drug success rates decay under heterogeneous spatio-environmental assump- tions}

In order to examine the effect that spatial heterogeneity of the ECNE concentrations and, thusly, the resulting cancer cell population on the longer term

effectiveness of targeted and immunological treatments, we considered only that treatment protocol which proved effective in the homogeneous case; namely that of an ipilimumab treatment followed by BRAFi post-treatment. The introduction of spatial heterogeneity whilst maintaining all other factors, in their en- 


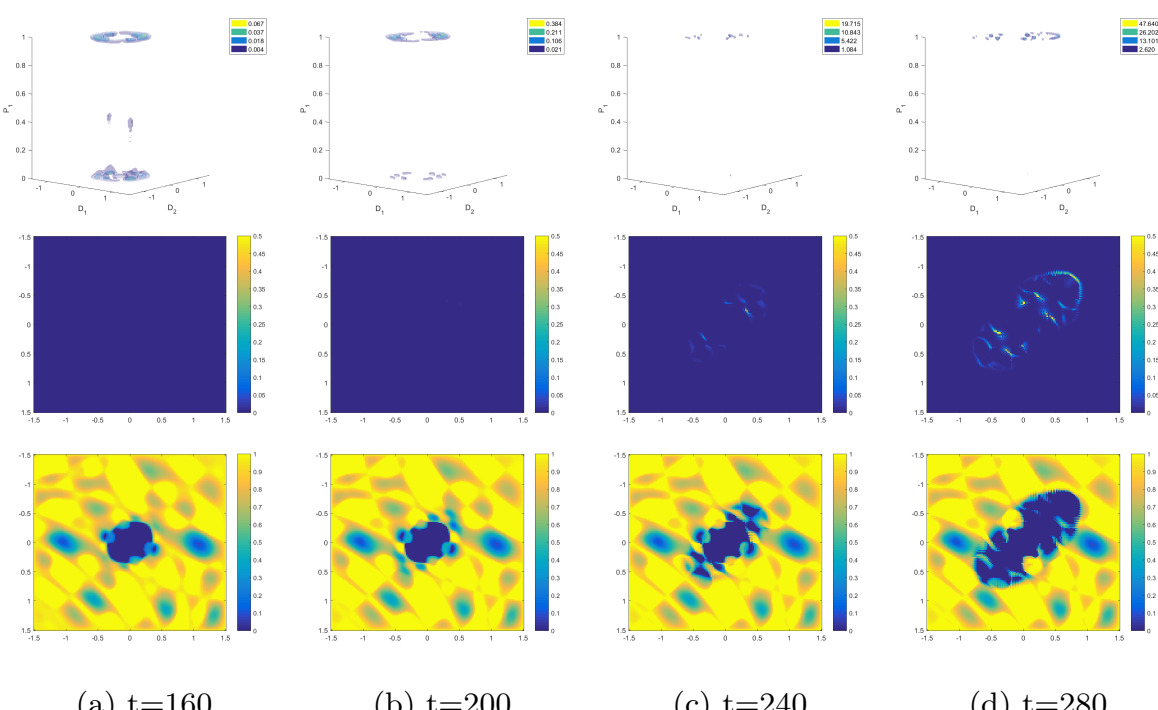
(a) $t=160$
(b) $t=200$
(c) $\mathrm{t}=240$
(d) $\mathrm{t}=280$

Figure 8: Spatial heterogeneity eradicates treatment success. Panels displaying (top) the structured cellular population with space across the lower plane and mutational state given along the vertical axis; (middle) the spatial cellular distribution; and (bottom) the ECNE density, where ipilimumab treatment is given at $t=40$ and BRAFi treatment is given at $t=100$, for time points $t \in\{160,200,240,280\}$ are shown.

tirety, was sufficient to cause the degeneration of treatment success into the characteristic death and regrowth curves seen previously (Results not shown, although they may be inferred from figures $7 \& 8$ middle).

Notice, firstly, that the spatial cancer cell population (Fig. $7 \& 8$ middle) initially spreads to the nearby regions of elevated ECNE concentration, prior to treatment. As the treatment is applied, and the regions of highest cell population coincide with the regions of highest ipilimumab concentration, the cell population is reduced to invisibility for some times $40<t<200$. It should be understood, here, that under a great evolutionary selective pressure only very few cells survive these initial waves of treatment but those cells which do survive will be completely resistant to both treatments. At this time, and with almost the entirety of the surviving cellular population being resistant to both BRAFi and ipilimumab, the cellular population begins to regrow at regions of highest 
nutritional content, or ECNE.

One may observe this dynamic in the spatio-structural cellular population, progressively over the entire time domain. Consistently with the punctuated equilibrium assumptions within the model, one notices a pulsatile movement of the cellular subpopulations between $y=0$ and $y=1 / 2$, and again towards $y=1$ (Fig. $7 \& 8$ top). In particular, however, the first time at which the cancer cell population has been visibly eradicated (Fig. 7c), the visible coincidence of those areas of low ECNE concentration with those cancer cell clusters at the most elevated value of $y$. In other words, the difference in the heterogeneous case, as compared with the homogeneous case, is that the cancer cell population is able to preferentially avoid drug-induced apoptosis by remaining in regions of low ECNE and drug concentrations, which allows the cellular population to become resistant before migrating to regions of high nutrition and increasing their collective proliferation rate.

This demonstrates that particular prudence must be paid during consideration of spatial factors in the study of drug resistance and strategy. One should also notice the clinically difficult tumour that results from this method of treatment (Fig. 8d middle) and the nature of the underlying environmental infrastructure, or ECNE. The tumour is viable although sparsely populated which raises significant questions about the ability to remove such a tumour, surgically. The approach to treating such a patient would classically be to use chemical means, which have now been exhausted and given rise to a uniformly resistant tumour.

625

\section{Metabolic Remodeling and the Re-Establishment of Drug Sensi- tivity}

Recent studies have looked at the effect of BRAFi on the human melanoma PDX lines implanted in the immunodeficient mouse and found that this drug is largely ineffective, implicating a role for the immune system in its functioning. This result is contrasted with the effectiveness at eradicating the tumour with 
BRAFi+MEKi, again with the characteristic relapse curve [66].

These same studies have suggested that after a primary phase of treatment, and subsequent washing of the drug species from the tumour, the cancerous cells

temporal concentration of BRAFi and we, now, redefine that $p_{2}(t, x)$ should 


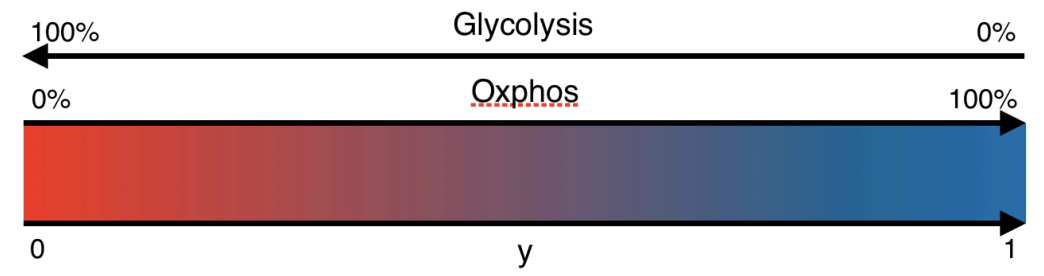

Figure 9: Visual reinterpretation of the structural $y$ variable to account for the metabolism of glucose molecules proportionally and competitively through glycolytic and oxphos pathways, respectively.

be given by the spatio-temporal concentration of MEKi, a second metabolic inhibitor of glycolysis.

\subsection{Re-interpreting the structural dimension for a metabolic system}

In order to capture the re-sensitisation phenomenon, we must reinterpret the structural $y$ variable to take into account the newfound plasticity of the cellular population. We assume that the effect of the drugs and the variability in the cellular population may be adequately illustrated through the cellular pathways involved in the metabolism of glucose; namely those of glycolysis and of oxphos. Given that a given glucose molecule, may be metabolised through the utilisation of either one of these pathways, but not both, we may represent the structure of the cell as the proportion of glucose sent to glycolytic pathways as opposed to oxphos pathways; such that $y=0$ represents $100 \%$ of glucose being metabolised through glycolysis, and $0 \%$ by oxphos, whilst $y=1$ represents $0 \%$ of glucose being metabolised through glycolysis, and $100 \%$ by oxphos (Fig. 9).

\subsection{A cellular growth function in a metabolic system}

Likewise with our previous paradigm, we assume that proliferation requires the presence of nutrients, $m_{2}(t, x)$, above a certain threshold, $\theta_{m_{2}}$. As was recognised by Warburg in 1956 [70], and was subsequently termed the Warburg effect, highly proliferative cancer cells appear to preferentially utilise glycolytic pathways to synthesise membrane lipids and other essential components from 
glucose. Therefore, we assume that there exists some underlying proliferation rate, $\phi_{c, 1}$, which is common amongst all cells and a further 'Warburg' proliferation rate, $\phi_{c, 2}$, which is contributed dependent upon the degree to which the cell utilises glycolysis; as the cell utilises the glycolytic pathways to a greater extent, its proliferation rate shall increase concurrently. Moreover, since we are particularly interested in the cell's ability to absorb and utilise available nutrients in the environment, we modify our competition assumptions so that the cellular population's proliferation will not be inhibited by the presence of the ECNE but will rather simply increase the pressure on the ECNE itself. Thusly, we replace the unoccupied volume term by $1-\int_{\mathcal{P}} c d y$ and write the full growth term as

$$
\phi_{M}(y, c, \bar{m}):=c\left(1-\int_{\mathcal{P}} c d y\right)\left(m_{2}-\theta_{m_{2}}\right)\left(\phi_{c, 1}+\phi_{c, 2}(1-y)\right) .
$$

\subsection{A structural flux function in a metabolic system}

The cell is biologically engineered to complete its cell cycle and evolution has selected for cellular populations who are particularly efficient at achieving this goal. Therefore, given that a cell requires nutrition and the ability to freely adapt in order to achieve this objective, if the cell is deprived of its essential environment then it will take extreme measures in order to continue to proliferate. We here define stress, or 'stressed conditions', as those conditions which are not conducive to cellular metabolism and proliferation. In particular, those scenarios which would lead the cell to feel 'stressed' are given explicitly by nutritional deprivation or targeted inhibition of metabolically essential genes, such as BRAF or MEK. Therefore, we define the weighted stress term as $\psi_{p_{1}} p_{1}+\psi_{p_{2}} p_{2}-\psi_{m_{2}} m_{2}$, where $\psi_{p_{1}}, \psi_{p_{2}}, \psi_{m_{2}}$, are positive weights such that $\psi_{p_{1}}+\psi_{p_{2}}+\psi_{m_{2}}=1$. Under stress, the cell shall randomly diversify its behaviour; each cell becoming stochastically more or less oriented towards glycolytic metabolism such that the population, as a whole, becomes more metabolically diverse. Therefore, we may represent this at the population level by a structurally diffusive behaviour. The 


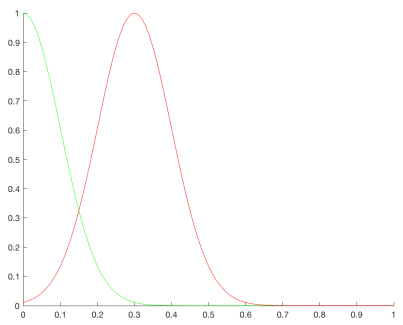

Figure 10: Distributions for the drug effectiveness functions $\mathfrak{f}_{1}(y)($ green $)$ and $\mathfrak{f}_{2}(y)(r e d)$.

structural diffusion coefficient $\Sigma(y, \bar{m}, \bar{p})$ is proportional to the weighted stress, therefore

$$
\Sigma(y, \bar{m}, \bar{p})=\sigma_{c}\left(\psi_{p_{1}} p_{1}+\psi_{p_{2}} p_{2}-\psi_{m_{2}} m_{2}\right)
$$

715

where $\sigma_{c}$ is a positive constant. In the absence of stress, the cell population relaxes by advection to the preferential metabolic state $y=\omega_{c}$. The relaxation rate is proportional to the weighed non-stressed factor defined as $1-\psi_{p_{1}} p_{1}-$ $\psi_{p_{2}} p_{2}$. Thus, the normalized structural velocity reads

$$
\Psi_{M}(y, \bar{m}, \bar{p})=\bar{\sigma}_{c}\left(\omega_{c}-y\right)\left(1-\psi_{p_{1}} p_{1}-\psi_{p_{2}} p_{2}\right)
$$

where $\bar{\sigma}_{c}$ is a positive constant.

720

\subsection{Drug effectiveness functions in a metabolic system}

The drug effectiveness functions for BRAFi and MEKi, $p_{1}(t, x)$ and $p_{2}(t, x)$ respectively (further discussion in Appendix A.2), are given simply by Gaussian functions centred at $\alpha_{\mathfrak{f}_{1}}=0$ and $\alpha_{\mathfrak{f}_{2}}=3 / 10$ respectively. We write these 725 mathematically

$$
\left\{\begin{array}{l}
\mathfrak{f}_{1}(y):=\exp \left[-\beta_{\mathfrak{f}_{1}}\left(y-\alpha_{\mathfrak{f}_{1}}\right)^{2}\right] \\
\mathfrak{f}_{2}(y):=\exp \left[-\beta_{\mathfrak{f}_{2}}\left(y-\alpha_{\mathfrak{f}_{2}}\right)^{2}\right] .
\end{array}\right.
$$

whilst the widths of these Gaussian functions are uniform with $\beta_{\mathfrak{f}_{1}}=\beta_{\mathfrak{f}_{2}}=50$ 
(Fig. 10), in order to replicate results from the murine models from [66].

\section{Results for the Metabolic System}

Again, our primary motivating factor for these metabolically plastic systems ing or predicting the complex dynamics underlying in vivo results (Section 6.1). Beyond this, we wished to try to understand the spatio-metabolic dynamics of the tumour which are allowing resistance to develop (Section 6.2). Finally, given the complexity of the plastic model, we wished to know what the dynamics of which we selected as $t=210$ in our tumours. A final dose was given after approximately 30 days of unimpeded growth, at $t=240$.

\subsection{Resistance and re-sensitisation dynamics are captured by plastic, metabolic} in silico modelling

As is the case with the in vivo experiments, we observe the death, tolerance, and regrowth pattern within the tumour (Fig. 11). This is then followed by a period of rapid, unimpeded growth due to the removal of drugs from the tumour. It is important to notice that upon the second wave of treatment, the tumour is 755 again eradicated entirely for some brief period before becoming resistant more 


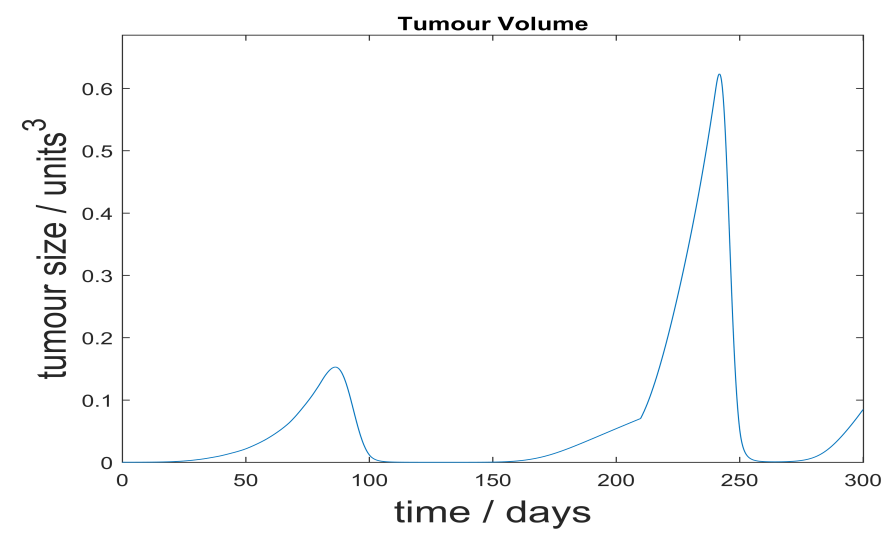

Figure 11: Resistance and re-sensitisation dynamics are captured by in silico modelling. Graph displaying the tumour volume of the metabolic tumour model over the duration of the in silico experiment, with continuous doses given from $t \in\{80,240\}$ and a drug holiday initiated at $t=210$

rapidly on this second occasion (Fig. 11). This correlates qualitatively with the in vivo results but may not be explained by a mutational model since those resistant cells would not reestablish their sensitivity to treatment. This effect is termed 're-sensitisation' and may be biologically and clinically significant.

In order to more accurately capture the results of the biological, experimental approach we use a lower dosing rate in this model. Also, the dose was applied uniformly in time between the start and the end of the treatment, instead of instantaneously (we used Heaviside functions instead of Dirac functions for the drug temporal profiles). This ensured a more gradual switch from the initial growth stage in the tumour to a drug-sensitive apoptotic phase, prior to tolerance (Fig. 11). Moreover, the primary regrowth stage appears to be damped in comparison to the mutational model under BRAFi treatment, alone, but this could be explained by the supplementary dosing of the tumour with MEKi, stunting regrowth to a greater extent. 

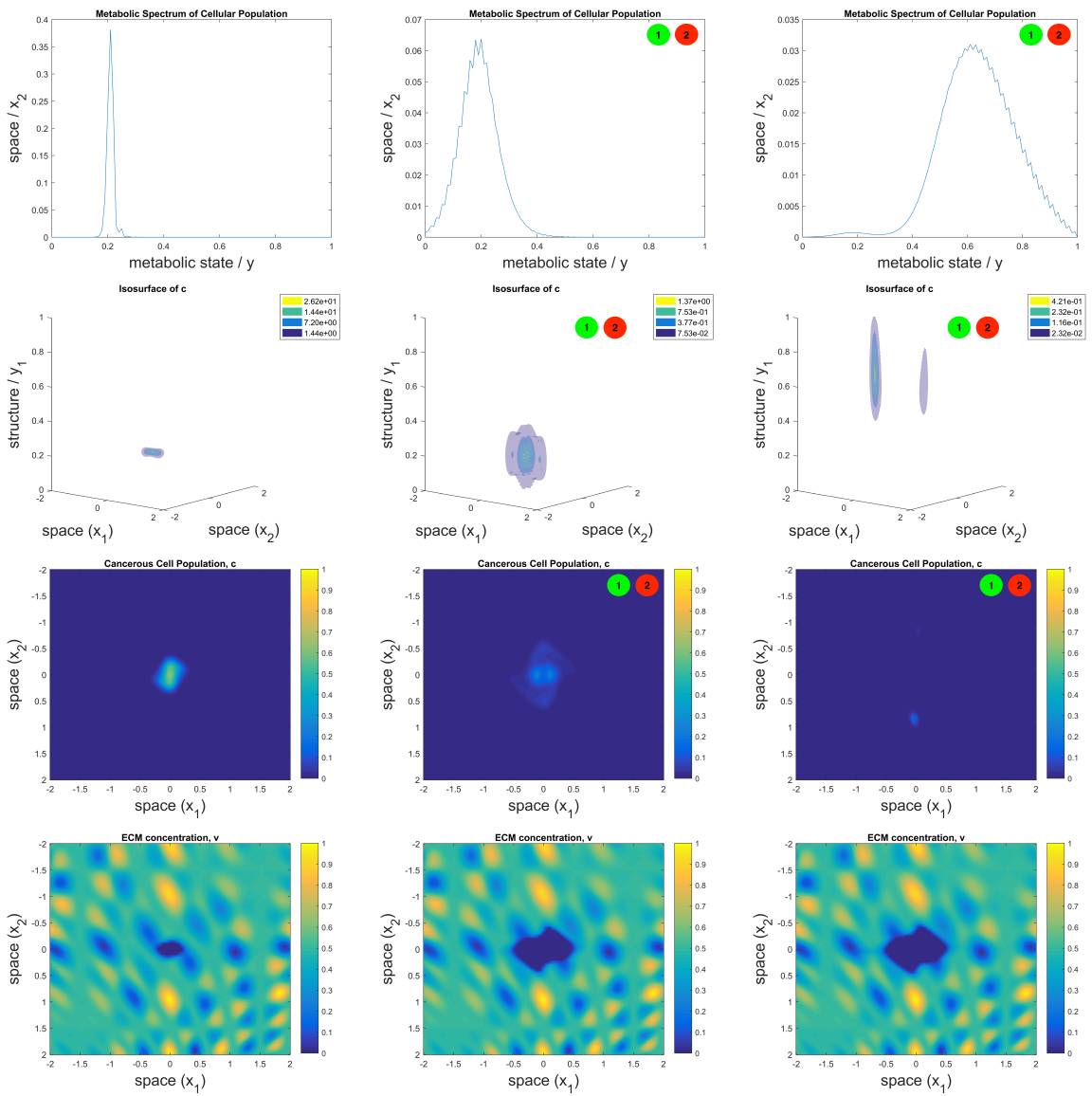

(a) $\mathrm{t}=50$

(b) $\mathrm{t}=100$

(c) $\mathrm{t}=150$

Figure 12: Tumours use oxphos metabolic pathways to resist targeted inhibition of glycolytic pathways by BRAFi and MEKi therapies. Shown are the phenotypic distribution (1 $1^{\text {st }}$ row); the spatio-phenotypic surface distributions $\left(2^{\text {nd }}\right.$ row); and spatial distribution $\left(3^{\text {rd }}\right.$ row) of the cellular population. The spatial distribution of the ECNE, with colour-bar, is also shown $\left(4^{\text {th }}\right.$ row), for completeness and in order that one can place the tumour within its environmental context. All figures are given at times $t \in\{50,100,150\}$ within subfigures (a), (b), and (c) respectively. Within the surface plots, the colours represent surfaces of approximately equal concentrations within the spatio-phenotypic context of the cell gradiated from lowest to highest concentration as purple, blue, green, then yellow.

6.2. Temporary oxphos metabolism may allow cancers to evade targeted treatments

Recall that lower values in $y$ are associated with more glycolytic modes of 

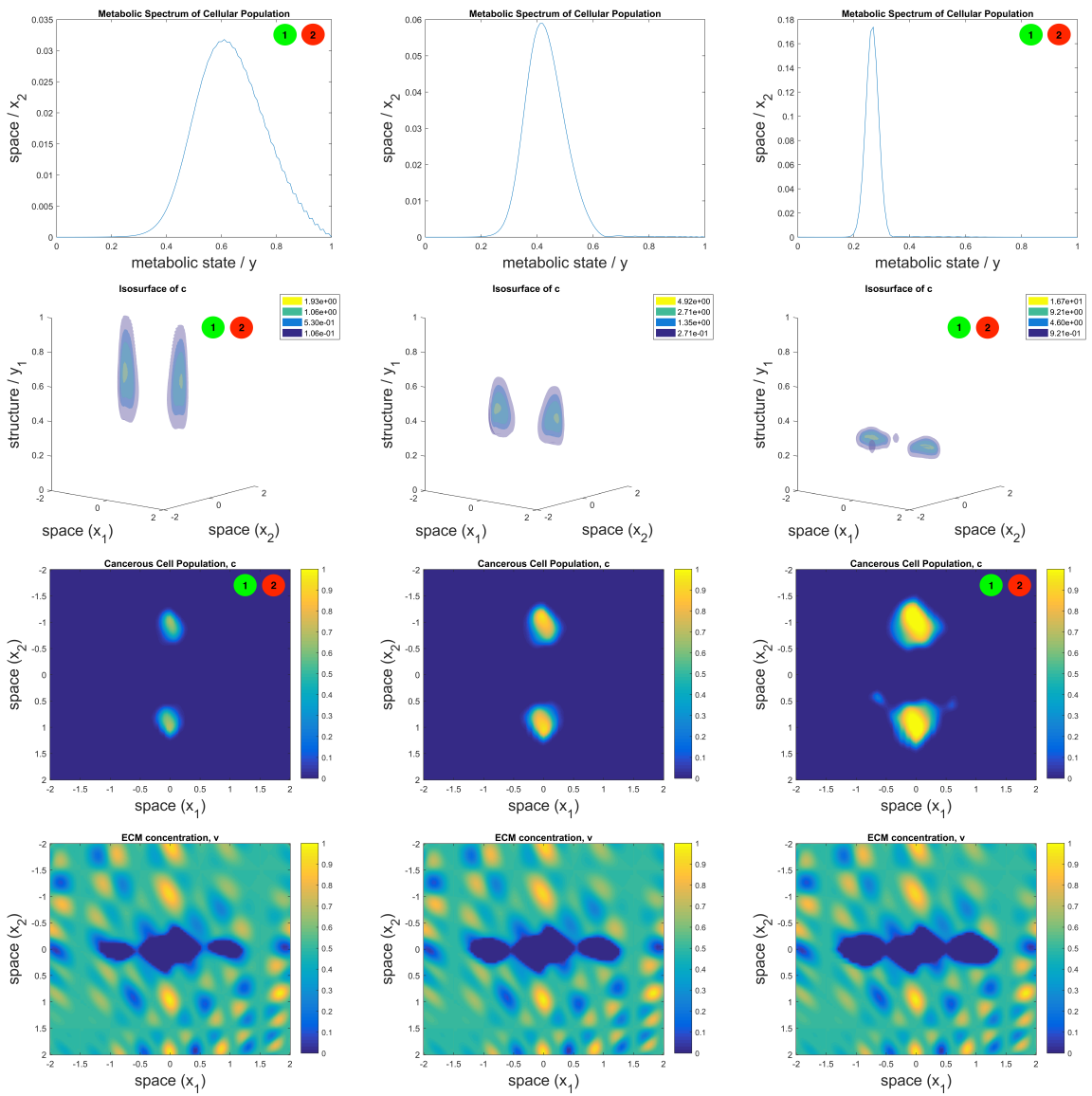

(a) $t=200$

(b) $t=220$

(c) $\mathrm{t}=240$

Figure 13: Tumours use oxphos metabolic pathways to resist targeted inhibition of glycolytic pathways by BRAFi and MEKi therapies. Shown are the phenotypic distribution ( $1^{\text {st }}$ row); the spatio-phenotypic surface distributions ( $2^{\text {nd }}$ row); and spatial distribution ( $3^{\text {rd }}$ row) of the cellular population. The spatial distribution of the ECNE, with colour-bar, is also shown ( $4^{\text {th }}$ row), for completeness and in order that one can place the tumour within its environmental context. All figures are given at times $t \in\{200,220,240\}$ within subfigures (a), (b), and (c) respectively. Within the surface plots, the colours represent surfaces of approximately equal concentrations within the spatio-phenotypic context of the cell gradiated from lowest to highest concentration as purple, blue, green, then yellow.

metabolism, where higher values of $y$ are associated with more oxphos modes of metabolism and that each of these structural $y$-coordinates is associated with a 

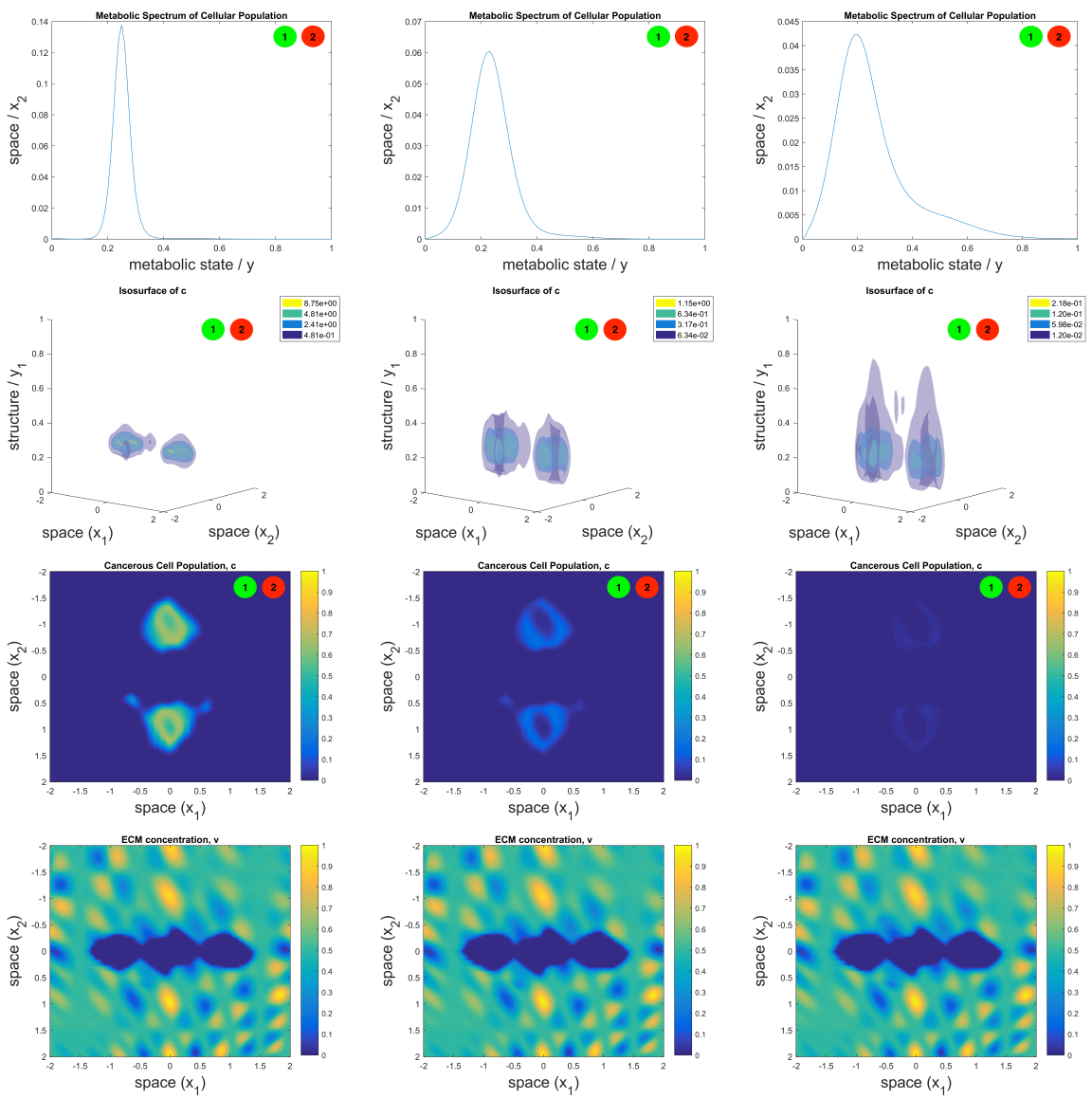

(a) $\mathrm{t}=245$

(b) $\mathrm{t}=250$

(c) $\mathrm{t}=255$

Figure 14: Tumours use oxphos metabolic pathways to resist targeted inhibition of glycolytic pathways by BRAFi and MEKi therapies. Shown are the phenotypic distribution ( $1^{\text {st }}$ row); the spatio-phenotypic surface distributions ( $2^{\text {nd }}$ row); and spatial distribution ( $3^{\text {rd }}$ row) of the cellular population. The spatial distribution of the ECNE, with colour-bar, is also shown ( $4^{\text {th }}$ row), for completeness and in order that one can place the tumour within its environmental context. All figures are given at times $t \in\{245,250,255\}$ within subfigures (a), (b), and (c) respectively. Within the surface plots, the colours represent surfaces of approximately equal concentrations within the spatio-phenotypic context of the cell gradiated from lowest to highest concentration as purple, blue, green, then yellow.

2D spatial $x$-coordinate. Moreover, a green encircled 1 in the upper right-hand corner of a graphic shall signify that the tumour is under BRAFi treatment, 

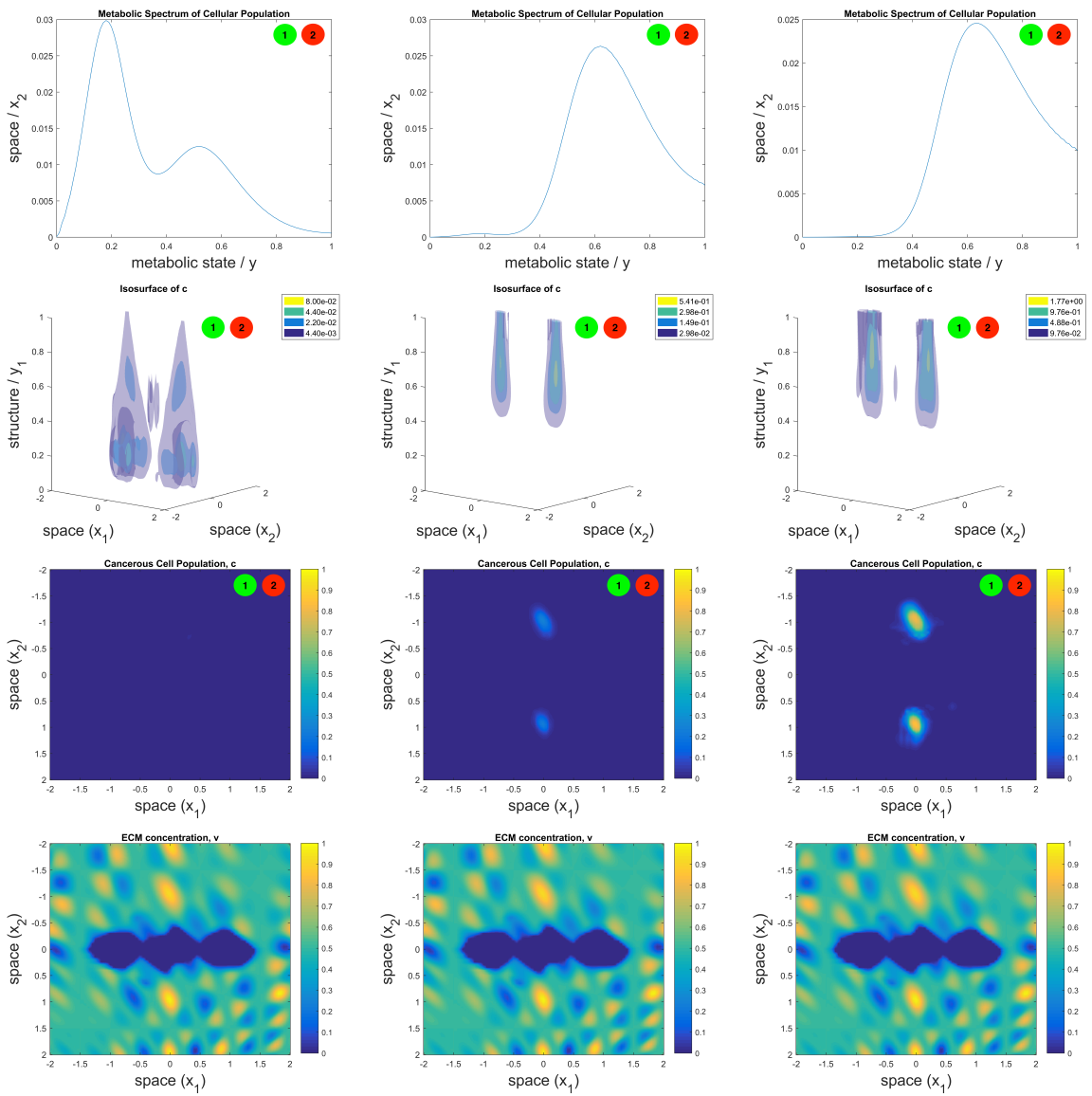

(a) $\mathrm{t}=260$

(b) $\mathrm{t}=280$

(c) $t=300$

Figure 15: Tumours use oxphos metabolic pathways to resist targeted inhibition of glycolytic pathways by BRAFi and MEKi therapies. Shown are the phenotypic distribution ( $1^{\text {st }}$ row); the spatio-phenotypic surface distributions ( $2^{\text {nd }}$ row); and spatial distribution ( $3^{\text {rd }}$ row) of the cellular population. The spatial distribution of the ECNE, with colour-bar, is also shown ( $4^{\text {th }}$ row), for completeness and in order that one can place the tumour within its environmental context. All figures are given at times $t \in\{260,280,300\}$ within subfigures (a), (b), and (c) respectively. Within the surface plots, the colours represent surfaces of approximately equal concentrations within the spatio-phenotypic context of the cell gradiated from lowest to highest concentration as purple, blue, green, then yellow.

where a red encircled 2 in the upper right-hand corner of a graphic shall signify that the tumour is under MEKi treatment (Fig. 12-15). 
tion is tightly associated with a glycolytic metabolic state and that its spatial composition is compact, whilst during the sensitivity phase (Fig. 12b) the cell population begins to diverge from this behaviour and cells may be spatially observed further afield. Moreover, and throughout this phase, one can observe the degeneration of the narrow peak, during the initial growth phase (Fig. 12a, $1^{\text {st }}$ row), into a larger metabolic distribution centred at the same position as this initial peak (Fig. 12b, $1^{\text {st }}$ row). The increase in variance of the metabolic distribution is as a result of the diversification of metabolism under stressed conditions, whereas the displacement of the mean towards a resistant oxphos population (Fig. 12c, $1^{\text {st }}$ row) is as a result of selective pressure.

During the resistance phase, the newly oxphos population continues to proliferate (Fig. 13a), whilst any glycolytic cells are induced to apoptosis. When the drugs are washed from the tumour, however, at $t=210$ one observes the cellular population beginning to migrate monotonically towards its preferred metabolic state (Fig. 13b, $1^{\text {st }} \& 2^{\text {nd }}$ rows), $\omega_{c}$ as observed at earlier time points (Fig. 12a, $1^{\text {st }}$ row), before reestablishing its glycolytic phenotype $y \approx \omega_{c}=0.2$ at $t=240$ (Fig. 13c). This whole process is then repeated during the second wave of treatment (Fig. 13c, $14 \& 15$ ), with the tumour being visibly eradicated during a process of metabolic diversification and upheaval (Fig. 14b, 14c \& 15a) before regrowing as an oxphos oriented tumour (Fig. 15b \& 15c).

In this model, one may far more clearly see that the regrowth in the tumour is spatially correlated with the regions of highest ECNE concentrations (Fig. $12 \mathrm{c}, 3^{\text {rd }} \& 4^{\text {th }}$ rows) and those regions where the cellular species will necessarily have the greatest access to nutrients. Interestingly, this will also be the spatial subregion in which the selective pressure is most elevated due to the presence

Observe, then, that in the initial growth phase (Fig. 12a) the cell populaof high concentrations of BRAFi+MEKi leading to the apoptosis of glycolytic cells and selecting for a more oxphos-dependent population of cells (Fig. 12c \& $13 \mathrm{a}, 2^{\text {nd }}$ row).

To sum the above analysis of these results, the tumour exhibits an initially glycolytic mode of metabolism which, through stress-induced diversification, de- 
cays into a less defined mode of glucose metabolism. By spatially correlating with regions of heightened nutritional content, these resistant oxphos cells are able to outgrow their drug-induced apoptotic rate and proliferate. By removing the drug from the tumour, and the stressor of the cell, the cellular population ultimately allowing the second wave of treatment to visibly eradicate the remaining population of cells. Nevertheless, these cells are able to regain their metabolic advantage and return to an oxphos state, in order to once again become resistant to treatment.

820

6.3. More rapid secondary resistance wave may be explained by residual oxphos populations

One feature of the growth, which is of great clinical significance, is that of the increased rapidity to resistance upon the second wave of treatment (Fig. 11). cancer cell population, towards the preferred glycolytic state, during the drug holiday (Fig. 13). The tail on the right-hand side of the oxphos cell distribution (Fig. 13a \& 13b, $1^{\text {st }}$ row) are not entirely consolidated during their backwards migration but, rather, remain as a residual oxphos cell population (Fig. 13b, ${ }_{830} 1^{\text {st }}$ row), which begin to appear upon selective degradation of glycolytic populations (Fig. 14b, $1^{\text {st }}$ row). Although these cells will migrate gradually towards their preferred metabolic state, $\omega_{c}$, it could be that their lower local nutritional value is allowing them to retain their oxphos state to a greater extent than the remainder of the population. Under the selective pressure applied by the drug, the glycolytic subpopulation is degraded, as it again attempts to diversify its metabolic status, whilst the oxphos population is free to grow (Fig. 14b, 14c, \& 15,1 st $\& 2^{\text {nd }}$ rows), eventually replacing the glycolytic population as the dominant population within the tumour (Fig. 15b \& 15c).

One may also clearly observe the difference in the spatio-structural distri840 butions 20 days posterior to the first wave of treatment (Fig. 12b, $2^{\text {nd }}$ row) in comparison to 20 days posterior to the second (Fig. 15a, $2^{\text {nd }}$ row). After the 
first wave of treatment, the tumour having never been exposed to stress prior to this event, the metabolic profile of the tumour is neatly distributed around its preferred glycolytic state. After the second wave of treatment, however, the metabolic profile is bimodal, with a distinct oxphos as well as a glycolytic population. This appears to be due to the fact that not all of the cells from the resistant oxphos population have migrated fully back to their preferred glycolytic state and are, thus, able to repopulate the new resistant population far more rapidly since they are not subject to the same selective pressures as their glycolytic counterparts.

\section{Discussion}

We have introduced a general modelling framework for evolution of heterogeneity in solid tumours submitted to multiple drug therapy, wherein the definition of an appropriate normalized structural velocity, $\Psi(y, \bar{m}, \bar{p})$; structural diffusion matrix, $\Sigma(y, \bar{m}, \bar{p})$; growth function, $\Phi_{c}(y, \bar{m}, c, v)$; and vector valued drug effectiveness function, $\overline{\mathfrak{f}}(y)$, may give rise to importantly nuanced patterns of behaviour. Using this framework, we then introduced two primary models for considering different dynamics within a tumour population. Firstly, the mutational model considered population level dynamics for a system in which an individual cell will sequentially undergo a BRAF mutation, followed by subsequent mutations which confer resistance to BRAFi and ipilimumab therapies. Secondly, we considered a plastic model of drug resistance, in which the switching of cellular dependence on glycolytic and oxphos pathways for the metabolism of glucose may confer a survival advantage when faced with glycolysis inhibiting BRAFi+MEKi treatments.

Using our mutational model to consider paradigms of punctuated equilibrium and phyletic gradualism in the evolution of the cellular genome, we found that punctuated equilibrium assumptions were more consistent with biological data. This shows good consistency with the modern cancer genomic literature, in asserting that short term catastrophes, rather than the gradual accumulation of mutations, is more likely to contribute to the mutational state of tumours 
$[47,48,49]$. We also predicted that using ipilimumab, immune cell-enhancers, in advance of a BRAFi is more effective at reducing the tumour population over the long term. This model prediction is confirmed by studies which used both

these subpopulations. These metabolically resistant populations will then preferentially re-sensitise themselves through metabolic remodeling, allowing for the 
effective second wave of treatment.

Moreover, our model provides an opportunity to understand the underlying dynamics of such metabolically plastic tumours and also the mechanisms of resistance and re-sensitisation, showing strong agreement with in vivo PDX tumour experiments. For both waves of treatment, our model shows a characteristic death, tolerance, and regrowth pattern, but with a quicker relapse occurring with the second wave of treatment. Experiments conducted by Rambow et al. [66] also show this pattern of death and growth, with faster regrowth posterior to the second wave of treatment, such that our model may provide an explanation of this phenomenon. Residual, metabolically resistant cells from the first wave of treatment may provide a basis for a resistant population to grow back more quickly upon the second wave of treatment. Implicitly, our model would predict that reducing treatment to as great an extent as is possible, whilst still eradicating the tumour, would reduce the opportunity for the tumour to establish this residual population and resist future waves of treatment.

\section{Appendix A. Thorough Discussion and Justification of the Mathe- matical Model}

Current modelling approaches consider the cell as a discretely changing variable who exists in an explicitly sensitive or resistant state. We wish, here, to conceive of the cell as a continuously changing and finely tunable evolutionary population. Different cancer cells have similar, if not identical, origins and are not innately differentiable but have rather gained different characteristics. Therefore, we introduce a novel modelling framework in order to reconceive the mathematical representation of the cell, from this more nuanced perspective.

Cells do, however, function differently. Within these categories, then, there must be a wealth of diversity to reflect the reality of the structural differences between cells. In order to reflect this, we incorporate a term that operates similarly to those structural models previously employed $[5,6]$, whilst building 
on the solid mathematical derivation given by existing spatio-structuro-temporal models $[9,11]$. Letting $\mathcal{I}:=[0, T] \subset \mathbb{R}_{+}$be the time interval over which the experiment is conducted; $\mathcal{D}:=[0,1]^{2} \subset \mathbb{R}_{+}^{2}$ be the spatial domain; and $\mathcal{P}:=$ ${ }_{935}[0,1] \subset \mathbb{R}_{+}$define the continuous domain over which the mutational or metabolic changes may occur, we couple these dynamics using a simple conservation of mass assumption. If $V \times W \subseteq \mathcal{D} \times \mathcal{P}$ is an arbitrary volume of the spatiostructural domain with piecewise smooth boundaries $\partial V$ and $\partial W$ respectively, then we can write that the total population of cells in this volume is given by

$$
c(t)_{V \times W}=\int_{W} \int_{V} c(t, x, y) d x d y
$$

Then we can use an existing mathematical framework $[9,11]$ to deduce that the change in cell density $c(t, x, y)$ is given by the partial differential equation

$$
\frac{\partial}{\partial t} c(t, x, y)=\nabla_{x} \cdot \underbrace{F(c, v, \bar{m})}_{\text {Spatial Flux }}+\nabla_{y} \underbrace{\mathrm{G}(y, c, v, \bar{m}, \bar{p})}_{\text {Structural Flux }}+\underbrace{S(y, c, v, \bar{m}, \bar{p})}_{\text {Source }} .
$$

Let $\Psi(y, \bar{m}, \bar{p}): \mathcal{I} \times \mathcal{D} \times \mathcal{P} \rightarrow \mathbb{R}$ be the normalized structural velocity for the cellular population. During a time interval of small length $\Delta t$, those cells having the mutational or metabolic state $y$ initially at $t$, will evolve to a state $945 y+r_{\mu} \Psi(y, \bar{m}, \bar{p}) \Delta t$ at $t+\Delta t$, where $r_{\mu}$ is the mean mutation rate. Moreover, let $\Sigma(y, \bar{m}, \bar{p}): \mathcal{I} \times \mathcal{D} \times \mathcal{P}$ be the structural diffusion matrix for the cellular population. Hence, the structural flux reads

$$
\begin{aligned}
G(y, c, v, \bar{m}, \bar{p}) & =G_{a}(y, c, v, \bar{m}, \bar{p})+G_{d}(y, c, v, \bar{m}, \bar{p}), \\
G_{a}(y, c, v, \bar{m}, \bar{p}) & =r_{\mu} \Psi(y, \bar{m}, \bar{p}) c(t, x, y), \\
G_{d}(y, c, v, \bar{m}, \bar{p}) & =-\Sigma(y, \bar{m}, \bar{p}) \nabla_{y} c(t, x, y) .
\end{aligned}
$$

With this concept of a continuum of phenotypic progression, we then recognise that pharmaceuticals are generally targeted at specific metabolic pathways 950 (related to selected cancer-related phenotypes and their respectively triggered mechanisms). Therefore, we employ a description of a phenotypic 'spectrum' wherein cells may inhabit any point on that available spectrum in $y$. These drugs may then target specific regions on this spectrum which employ the molecular 
pathways inhibited by these drugs. For this we form an effectiveness vector $\overline{\mathfrak{f}}(y) \in \mathcal{Y}^{P}$ which describes the bandwidth in the mutational dimension $\mathcal{P}$ on which the drug is effective at diminishing the population of cells, for each given drug, $p_{j}, j \in\{0, \ldots, P\}$.

\section{Appendix A.1. Discussion on Single-Dosage Systems}

The choice of mutational rate of change function would have to accurately represent the most sensible possible case for PG and PE, respectively. From (A.3) it is clear that the no-flux boundary condition is fulfilled automatically if the structural veolocity satisfies

$$
\Psi(\tilde{y}, \bar{m}, \bar{p})=0, \quad \forall \tilde{y} \in \partial \mathcal{P}
$$

where $\partial \mathcal{P}$ is the boundary of the structural domain $\mathcal{P}$.

The structural velocity for PG is considered to be constant, except for a small region at the boundary. In order to construct such a function, we start with

$$
\hat{\Psi}_{g}(y, \bar{m}, \bar{p}):=1-\sum_{i=0}^{1}\left(1+\exp \left[-\beta_{g}\left((2 y-1) i-y+\frac{5}{\beta_{g}}\right)\right]\right)^{-1},
$$

where $\beta_{g}$ is chosen sufficiently large such that the function $\hat{\Psi}_{g}(y, \bar{m}, \bar{p})$ is close to one everywhere except at narrow neighborhoods of $y=0$ and of $y=1$. The symmetry of the function $\hat{\Psi}_{g}$ implies that no-flux boundary conditions can be achieved by the imposition of

$$
\Psi_{g}(y, \bar{m}, \bar{p}):=\psi_{g}\left(\hat{\Psi}_{g}(y, \bar{m}, \bar{p})-c_{g}\right)
$$

where the lower case $\psi_{g}$ gives the mutation rate parameter and $c_{g}:=\hat{\Psi}_{g}(0, \bar{m}, \bar{p})=$ $\hat{\Psi}_{g}(1, \bar{m}, \bar{p})$ (Fig. 2a).

For the PE function, one must consider several features. Beyond smoothness, that is needed for both technical and biological reasons, one must again satisfy 
the no-flux conditions and impose the further conditions

$$
\begin{aligned}
& \left.\frac{\partial^{2}}{\partial y^{2}} \Psi_{e}(y, \bar{m}, \bar{p})\right|_{y=\frac{1}{2}}>0,\left.\quad \frac{\partial^{2}}{\partial y^{2}} \Psi_{e}(y, \bar{m}, \bar{p})\right|_{y=\left\{\frac{1}{4}, \frac{3}{4}\right\}}<0, \\
& \left.\frac{\partial}{\partial y} \Psi_{e}(y, \bar{m}, \bar{p})\right|_{y=\left\{\frac{1}{4}, \frac{1}{2}, \frac{3}{4}\right\}}=0
\end{aligned}
$$

which is to say that maximal mutational velocity should occur between points of phyletic stability, "equilibria", and minimal velocity should occur at intermediate points of phyletic stability (where boundary conditions cover the cases of minimal and maximal phyletic deviance). Thus, one can choose a function of the form

$$
\hat{\Psi}_{e}(y, \bar{m}, \bar{p}):=\frac{1}{2}\left(1-\cos \left(2 \pi \hat{N}_{\psi} y\right)\right)+\alpha_{g} \sum_{i=1}^{N_{\psi}-1} \exp \left[-\beta_{e}\left(y-\frac{i}{N_{\psi}}\right)\right],
$$

where $N_{\psi}=3$ is the number of absolute mutational states in the considered paradigm (pre-mutated, BRAF mutated, \& resistantly mutated); $\beta_{e}$ is chosen such that distribution is increased smoothly; and the symmetry of this function in the domain implies that the no-flux boundary conditions can be satisfied by imposing

$$
\Psi_{e}(y, \bar{m}, \bar{p}):=\psi_{e}\left(\hat{\Psi}_{e}(y, \bar{m}, \bar{p})-c_{e}\right),
$$

where $\psi_{e}$ again gives the mutational rate and $c_{e}:=\hat{\Psi}_{e}(0, \bar{m}, \bar{p})=\hat{\Psi}_{e}(1, \bar{m}, \bar{p})$ (Fig. 2b).

Defining the cancerous population as being represented by a continuous distribution in the mutational space further allows one to define the drug effectiveness functions such that the drugs themselves target, not a discrete subset of the cellular population but rather, a continuous distribution of phenotypes which correspond to states in $\mathcal{P}$. With this in mind, we begin by defining the function itself as being represented by a vector of such distributions, each distribution describing the action of an individual drug

$$
\mathfrak{f}(y):=\left[\mathfrak{f}_{1}(y), \mathfrak{f}_{2}(y)\right]^{T} .
$$

Then, we can continue by defining the individual effectiveness functions of each 
drug as being given by the standard Gaussian distribution in $y$, such that

$$
\left\{\begin{array}{l}
\mathfrak{f}_{1}(y):=\exp \left[-\beta_{\mathfrak{f}_{1}}\left(y-\alpha_{\mathfrak{f}_{1}}\right)^{2}\right] \\
\mathfrak{f}_{2}(y):=\exp \left[-\beta_{\mathfrak{f}_{2}}\left(y-\alpha_{\mathfrak{f}_{2}}\right)^{2}\right],
\end{array}\right.
$$

where $\beta_{\mathfrak{f}_{1}}, \beta_{\mathfrak{f}_{2}}$ define, reciprocally, the breadth of the distribution and $\alpha_{\mathfrak{f}_{1}}, \alpha_{\mathfrak{f}_{2}}$ define the mutational position, in $y$, of maximal effectiveness.

In this case, since we consider $N_{\psi}=3$ mutationally-equidistant resting states for the tumour population, we define that the BRAF inhibitor, $p_{1}$, targets a mutational state which corresponds to the most susceptible of these resting (low relative mutational rate) states, $\alpha_{\mathfrak{f}_{1}}=\frac{1}{2}$. In this sense, also, we assert that the resting state, $\alpha_{\mathfrak{f}_{1}}$, represents a mutational state wherein the cell has a BRAF mutation, which makes cells in this state most susceptible to BRAFi treatment.

We then make a phenomenological choice, although with some support from the existing literature, to maximise the effectiveness of drug $p_{2}$, i.e. ipilimumab, at an arbitrary position between the establishment of mutational states corresponding to $y^{\prime} \in\left\{\frac{1}{2}, 1\right\}$, such that $\alpha_{\mathfrak{f}_{2}} \approx \frac{3}{4}$. We interpret the choice of $\alpha_{\mathfrak{f}_{1}}$ (smaller than $\alpha_{\mathfrak{f}_{2}}$ ) as conveying the sense that BRAFi is maximally responsive at early mutational states where PTEN mutation is developing but not established within the population, whereas ipilumab is maximally responsive at later stages. We assume this on the basis that treatment with BRAFi, prior to treatment with immune cell enhancers, is ineffective as opposed to the contrary and that this implies that sensitivity to BRAFi may occur at an early stage of mutational development.

\section{Appendix A.2. Discussion on Multi-Dosage Systems}

Thusly, we describe the metabolic change function, $\Psi: \mathcal{D} \times \mathcal{P} \rightarrow \mathbb{R}$, in terms of the phenotypic stress on the cell. We assume, firstly, that under a condition in which the influence of stressors is minimised, the cell has a preferred phenotypic state at $y=\omega_{c}$, which corresponds to a given utilisation of each pathway. We also assume that the primary stressors for the cell are malnutrition, which will 

act to deplete the cells ability to proliferate effectively.

Then the non-stressed term in the function must be given such that phenotypic advection is positive below this preferential state and negative above this state such that it will depend upon the relation $1-\omega_{c}$ for a non-dimensionalised system. The non-stressed condition must then be given by the opposing probability to that of stress such that $\Psi_{\bar{\sigma}}:=1-\psi_{p_{1}} p_{1}-\psi_{p_{2}} p_{2}$, with parameters chosen such that $\Psi_{\bar{\sigma}} \geq 0, \forall(t, x) \in \mathcal{I} \times \mathcal{D}$.

Stressed conditions for the cell are then quantified by the gradient of the cellular concentration in the region, giving a measurement of the collectivity of the behaviours of local cells. This choice of function for stressed conditions gives rise to diffusion under cellular stress, the rationale for which can be given by the intuitive understanding that cells diversify their behaviours in the presence of stressors. The magnitude of this stress is then determined by the concentrations of BRAFi, $p_{1}$, and MEKi, $p_{2}$, and is linearly diminished with the concentration of of nutrient species, $m_{2}$. All of these factors act as stressors to the cell and have their relative effects quantified by the weights $\psi_{p_{1}}, \psi_{p_{2}}, \psi_{m_{2}} \geq 0$, respectively. Then, the structural flux has diffusion and advection terms as follows

$$
\begin{aligned}
\Psi_{M}(y, \bar{m}, \bar{p}):= & -\sigma_{c}\left(\psi_{p_{1}} p_{1}+\psi_{p_{2}} p_{2}-\psi_{m_{2}} m_{2}\right) \nabla_{y} c(t, x, y) \\
& +\bar{\sigma}_{c}\left(\omega_{c}-y\right)\left(1-\psi_{p_{1}} p_{1}-\psi_{p_{2}} p_{2}\right) c(t, x, y),
\end{aligned}
$$

with the introduction of the stress, $\sigma_{c}$, and non-stress, $\bar{\sigma}_{c}$, parameters determining the weightings of the diffusion and advection terms with respect to one another.

Now, one must consider the nature and form of the effectiveness functions for the drug species, BRAFi $\left(p_{1}\right)$ and MEKi $\left(p_{2}\right)$, on the cellular population, in terms of their effect on the glycolytic or oxphos pathways. Firstly, we begin by writing the vector

$$
\mathfrak{f}(y):=\left[\mathfrak{f}_{1}(y), \mathfrak{f}_{2}(y)\right]^{T},
$$

to represent functions $\mathfrak{f}_{1}(y)$ and $\mathfrak{f}_{2}(y)$ in compact notation and begin by noticing that both of these drugs target genes essential to glycolysis. The transcription 
factors HIF1 $\alpha$, c-Myc, and Mondo A have been found to be downstream upregulators of glycolytic behaviours in $\mathrm{BRAF}^{v 600}$ cells [67, 71]. Moreover, BRAFi has been shown to prevent the hyperswitching of mutant melanoma cells to pyruvate based metabolism [72] - the primary product of glycolysis.

Withal, MEKi is responsible for targeting this same pathway, in melanoma cells. It has also been found that the PI3K pathway, activated by MEK, is responsible for glucose transport, and glycolytic metabolism, and can be inhibited by inhibition of MEK [73, 74].

The biological literature points to a link between melanoma associated genes, including BRAF and MEK, and the glycolytic pathway for glucose metabolism. Therefore, we write that the standard forms of the effectiveness functions will be Gaussian functions, with low values for variance, or high values for $\beta_{\mathfrak{f}_{1}}$ and $\beta_{\mathfrak{f}_{2}}$, such that

$$
\left\{\begin{array}{l}
\mathfrak{f}_{1}(y):=\exp \left[-\beta_{\mathfrak{f}_{1}}\left(y-\alpha_{\mathfrak{f}_{1}}\right)^{2}\right] \\
\mathfrak{f}_{2}(y):=\exp \left[-\beta_{\mathfrak{f}_{2}}\left(y-\alpha_{\mathfrak{f}_{2}}\right)^{2}\right] .
\end{array}\right.
$$

The values around which these functions are centred, $\alpha_{\mathfrak{f}_{1}}<\frac{1}{2}$ and $\alpha_{\mathfrak{f}_{2}}<\frac{1}{2}$, are chosen to align with the peak effect of the drug on the glycolytic and oxphos pathways.

Finally, we choose the proliferation function, $\phi_{M}: \mathcal{I} \times \mathcal{D} \times \mathcal{P} \rightarrow \mathbb{R}$, such that it is space-wise logistic in $c(t, x, y)$. Moreover, we assume that the cellular population requires nutrient in order to achieve positive proliferation and choose some arbitrary threshold value $\theta_{m_{2}}$ in order that, below such a value, the cellular population is depleted due to malnutrition. It is then imposed upon the system that there are two concurrent modes of proliferation: glycolytic and nonglycolytic. The non-glycolytic mode is not dependent upon the phenotypic state of the cell, $y$, and is rather an underlying process of all cells, whereas the glycolytic pathway is linearly enhanced by the percentage of glycolytic metabolism utilised (such that it is maximal at $y=0$ ). This is justified on account of the excess lipids produced through utilisation of glycolytic pathways. Therefore, we 


\begin{tabular}{|c|c|c|c|c|c|}
\hline & $c$ & $v$ & $m_{1}$ & $m_{2}$ & $p$ \\
\hline$D$ & $1 \times 10^{-5}$ & & $1 \times 10^{-4}$ & $1 \times 10^{-4}$ & $5 \times 10^{-4}$ \\
\hline$\phi$ & 0.4 & $5 \times 10^{-2}$ & 0.1 & 0.1 & \\
\hline$\delta$ & 2 & 10 & 0.1 & $5 \times 10^{-2}$ & $5 \times 10^{-2}$ \\
\hline$\chi$ & & $5 \times 10^{-5}$ & $1 \times 10^{-3}$ & 0 & 0 \\
\hline
\end{tabular}

Table B.1: List of parameters used for numerical simulations of the model. Parameters are defined within a non-dimensionalised system (excepting for time measured in days) and, as such, are defined in terms of units days ${ }^{-1}$.

write

$$
\phi_{M}(y, c, \bar{m}):=c\left(1-\int_{\mathcal{P}} c(t, x, y) d y\right)\left(m_{2}-\theta_{m_{2}}\right)\left(\phi_{c, 1}+\phi_{c, 2}[1-y]\right),
$$

1080

$c_{0}=\exp \left[-50\left(x^{2}+8 \cdot(y-\eta)^{2}\right)\right] \quad$ such that $\iint_{\mathcal{D}} \int_{\mathcal{P}} c_{0}(t, x, y) d x d y \approx 1 \times 10^{8}$,

where, since we know that the biological experiments were initiated with an approximate cell count of $2.5 \times 10^{4}$ cells, we assume that the cellular distribution is measured approximately in $10^{3}$ cells unit ${ }_{x}^{-2}$. Further, the default initial 
location in the phenotypic dimension is given by $\eta=\frac{1}{50}$. One should also clarify

given by a threshold of visibility $\theta_{v}$, we assume the proportionality of the tumour mass and the area of the section over which the tumour is visible, written as

$$
\iint_{\mathcal{D}} \int_{\theta_{y}}^{1} c(t, x, y) d y d x \approx k\left(\theta_{v}\right) \iint_{\mathcal{D}} \mathbb{1}\left\{\int_{\theta_{y}}^{1} c(t, x, y) d y \geq \theta_{v}\right\} \text { dx, }
$$

where the proportionality constant is dependent on the visibility threshold and is given by $k: \mathbb{R} \rightarrow \mathbb{R}$. To calculate the model's tumour volume, i.e. the volume of the tumour at $y \geq \theta_{y}$ and invoke the calculation from the tuning model [22] such that

$$
V_{c}:=K \sqrt{\left(\iint_{\mathcal{D}} \int_{\theta_{y}}^{1} c(t, x, y) d x d y\right)^{3}},
$$

with the adaptation of the ellipsoidal volume equation to $V=\frac{\pi}{6} a b \sqrt{a b}$ and where we take that $\theta_{y}=0.2$ and $K$ is an arbitrary constant. 
Then, in order to carry out our test experiment, we control the heterogeneity using the following formula for the initial condition

$$
c_{0}:=\sum_{j=1}^{J} \exp \left[-\left(x^{2}+\left(y-\eta_{j}\right)^{2}\right)\right], \quad \eta_{j} \in(0,0.5], \forall j \in\{1, \ldots, J\}
$$

and also in line with the initial volume condition (B.1), and where $J$ is in some sense a measure of the initial heterogeneity. We then apply the drug dosage periodically in time intervals given by $[0,20] \cup[40,60] \cup[80,100] \cup[120,140]$. For the simulations given in this current study, we use the range $J \in\{1, \ldots, 5\}$ to establish example data.

\section{Appendix B.2. Methods for Metabolic, Multi-Dosage Systems}

Due to the nature of the structural flux (A.9), it is necessary to develop a set of zero-flux boundary conditions which prevent, for example, diffusion in $y$ from causing cells to exit the domain, $\mathcal{P}$. Although (A.9) has both advection and diffusion terms, the metabolic change function is defined such that $\Psi_{\bar{\sigma}}(y)=0, y \in \partial \mathcal{P}$, meaning that advection fluxes are identically zero on the boundary. Therefore, we simply implement zero-Neumann boundary conditions on structural diffusion fluxes, namely $\nabla_{y} c(t, x, y)=0, y \in \partial \mathcal{P}$.

To begin treatment, one gradual dosage was given between $t=80$ and $t=100$, linearly in time, $t$. The drug was then washed from the tumour, in a step-wise fashion, at $t=210$, as this is the point at which the tumour volume had regrown to $\sim 20 \%$ of its previous maximum, and the tumour was allowed to regrow, unencumbered by glycolytic inhibitors for 30 days. A second gradual dosage was then given between $t=240$ and $t=260$, whereafter no further interventions were made.

Further, we define the unique structured population profile by the cellular population over the entirety of the spatial domain, $\mathcal{D}$, given by

$$
\hat{C}(t, y):=\iint_{\mathcal{D}} c(t, x, y) d \mathbf{x} .
$$

This can be used to describe the metabolic or structural profile of the tumour at a given time, $t$. 


\section{Acknowledgements}

The authors gratefully acknowledge financial support from the Canceropole GSO and École Doctorale I2S de l'Université de Montpellier. We are also grate${ }_{1145}$ ful to N. Theret, A. Devenyi, and D. Fisher for their critical reading of the manuscript.

\section{References}

[1] Humplik J, Hill AL, Nowak MA. Evolutionary dynamics of infectious diseases in finite populations. Journal of Theoretical Biology 2014;360:149-62.

[2] Ghang W, Nowak MA. Stochastic evolution of staying together. Journal of Theoretical Biology 2014;360:129-36.

[3] Bozic I, Nowak MA. Timing and heterogeneity of mutations associated with drug resistance in metastatic cancers. Proceedings of the National Academy of Sciences of the United States of America 2014;111(45):159648.

[4] Kleinman A. The mathematics of random mutation and natural selection for multiple simultaneous selection pressures and the evolution of antimicrobial drug resistance. Statistics in Medicine 2016;35(29):5391-400.

[5] Lorz A, Lorenzi T, Hochberg ME, Clairambault J, Perthame B. Populational adaptive evolution, chemotherapeutic resistance and multiple anticancer therapy. ESAIM: Mathematical Modelling and Numerical Analysis 2012;47:377-99.

[6] Lorz A, Lorenzi T, Clairambault J, Escargueil A, Perthame B. Modeling the Effects of Space Structure and Combination Therapies on Phenotypic Heterogeneity and Drug Resistance in Solid Tumors. Bulletin of Mathematical Biology 2015;77(1):1-22. 
[7] Eldredge N, Gould SJ. Models in Paleobiology; chap. 5. Punctuated Equilibria: An Alternative to Phyletic Gradualism. Freeman, Cooper and Company; 1972 , p. 82-115.

[8] Waclaw B. Evolution of drug resistance in bacteria. Advances and Medicine and Biology 2016;915:49-67.

[9] Domschke P, Trucu D, Gerisch A, Chaplain MAJ. Structured models of cell migration incorporating molecular binding processes. Journal of Mathematical Biology 2017;75(6-7):1517-61.

[10] Hodgkinson A, Chaplain MAJ, Domschke P, Trucu D. Computational approaches and analysis for a spatio-structural-temporal invasive carcinoma model. Bulletin of Mathematical Biology 2018;doi: \bibinfo\{doi\}\{https:// doi.org/10.1007/s11538-018-0396-4\}.

[11] Hodgkinson A, Radulescu O, Uzé G, Trucu D. Signal propagation in sensing and reciprocating cellular systems with spatial and structural heterogeneity. Bulletin of Mathematical Biology 2018;:(accepted).

[12] Gatenby RA, Gawlinski ET. A Reaction-Diffusion Model of Cancer Invasion. Cancer Res 1996;56(24):5745-53.

[13] Chaplain MAJ, Lolas G. Mathematical modelling of cancer invasion of tissue: Dynamic heterogeneity. Networks and Heterogeneous Media 2006;1(3):399-439.

[14] Jang S, Atkins MB. Which drug, And when, For patients with BRAFmutant melanoma? The Lancet Oncology 2013;14(2):e60-9.

[15] Bollag G, Hirth P, Tsai J, Zhang J, Ibrahim PN, Cho H, et al. Clinical efficacy of a RAF inhibitor needs broad target blockade in BRAF-mutant melanoma. Nature 2010;467:596-9.

[16] Paraiso KHT, Xiang Y, Rebecca VW, Abel EV, Chen YA, Munko AC, et al. PTEN loss confers BRAF inhibitor resistance to melanoma cells through the suppression of BIM expression. Cancer Research 2011;71(7):2750-60. 
[17] Paraiso KH, Fedorenko IV, Cantini LP, Munko AC, Hall M, Sondak VK, et al. Recovery of phospho-ERK activity allows melanoma cells to escape from BRAF inhibitor therapy. Br J Cancer 2010;102(12):1724-30.

[18] Rubinstein JC, Sznol M, Pavlick AC, Ariyan S, Cheng E, Bacchiocchi A, et al. Incidence of the V600K mutation among melanoma patients with BRAF mutations, and potential therapeutic response to the specific BRAF inhibitor PLX4032. Journal of translational medicine 2010;8:67.

[19] Smalley KSM, Lioni M, Dalla Palma M, Xiao M, Desai B, Egyhazi S, et al. Increased cyclin D1 expression can mediate BRAF inhibitor resistance in BRAF V600E-mutated melanomas. Mol Cancer Ther 2008;7(9):2876-83.

[20] Martz CA, Ottina KA, Singleton KR, Jasper JS, Wardell SE, PerazaPenton A, et al. Systematic identification of signaling pathways with potential to confer anticancer drug resistance. Science Signalling 2015;57(6):74268.

[21] Graziani G, Artuso S, De Luca A, Muzi A, Rotili D, Scimeca M, et al. A new water soluble MAPK activator exerts antitumor activity in melanoma cells resistant to the BRAF inhibitor vemurafenib. Biochemical Pharmacology 2014;95(1):16-27.

[22] Perna D, Karreth FA, Rust AG, Perez-Mancera PA, Rashid M, Iorio F, et al. BRAF inhibitor resistance mediated by the AKT pathway in an oncogenic BRAF mouse melanoma model. Proceedings of the National Academy of Sciences of the United States of America 2015;112(6):E53645.

[23] Villanueva J, Infante JR, Krepler C, Reyes-Uribe P, Samanta M, Chen HY, et al. Concurrent MEK2 Mutation and BRAF Amplification Confer Resistance to BRAF and MEK Inhibitors in Melanoma. Cell Reports 2013;4(6):1090-9. 
[24] Shi H, Hong A, Kong X, Koya RC, Song C, Moriceau G, et al. A novel AKT1 mutant amplifies an adaptive melanoma response to BRAF inhibition. Cancer Discovery 2014;4(1):69-79.

[28] Nazarian R, Shi H, Wang Q, Kong X, Koya RC, Lee H, et al. Melanomas acquire resistance to b-raf(v600e) inhibition by rtk or n-ras upregulation. Nature 2010;468:973-7.

[29] Alcalá AM, Flaherty KT. BRAF inhibitors for the treatment of metastatic

[30] Slipicevic A, Holm R, Nguyen MTP, Bøhler PJ, Davidson B, Flørenes VA. Expression of activated Akt and PTEN in malignant Melanomas: Relationship with clinical outcome. American Journal of Clinical Pathology 1245

[31] Kamo N, Ke B, Busuttil RW, Kupiec-Weglinski JW. PTEN-mediated akt/b-Catenin/foxo1 signaling regulates innate immune responses in mouse liver ischemia/reperfusion injury. Hepatology 2013;57(1):289-98. 
[32] Ouyang W, Beckett O, Flavell RA, Li MO. An Essential Role of the Forkhead-Box Transcription Factor Foxo1 in Control of T Cell Homeostasis and Tolerance. Immunity 2009;30(3):358-71.

[33] Kim M, Ouyang W, Liao W, Zhang M, Li M. The transcription factor foxo1 controls central-memory $\mathrm{CD} 8+\mathrm{T}$ cell responses to infection. Immunity 2013;39(2):286-97.

[34] Hess Michelini R, Doedens AL, Goldrath AW, Hedrick SM. Differentiation of CD8 memory $\mathrm{T}$ cells depends on Foxo1. The Journal of experimental medicine 2013;210(6):1189-200.

[35] Günzl P, Schabbauer G. Recent advances in the genetic analysis of PTEN and PI3K innate immune properties. Immunobiology 2008;213(9-10):75965 .

[36] Davies H, Bignell GR, Cox C, Stephens P, Edkins S, Clegg S, et al. Mutations of the BRAF gene in human cancer. Nature 2002;417(6892):949-54.

[37] Chapman PB, Hauschild A, Robert C, Haanen JB, Ascierto P, Larkin J, et al. Improved survival with vemurafenib in melanoma with BRAF V600E mutation. N Eng J Med 2011;364(26):2507-16.

[38] Goel VK, Lazar AJF, Warneke CL, Redston MS, Haluska FG. Examination of mutations in BRAF, NRAS, and PTEN in primary cutaneous melanoma. J Invest Dermatol 2006;126(1):154-60.

[39] Darwin C. On the Origin of Species by Means of Natural Selection. John Murray; 1859.

[40] Dawkins R, Krebs JR. Arms races between and within species. Proceedings of the Royal Society B 1979;205(1161).

[41] Mayr E. Change of genetic environment and evolution. 1954. 
[42] Lieberman BS, Eldredge N. What is punctuated equilibrium? What is macroevolution? A response to Pennell et al. Trends in Ecology and Evolution 2014;29(4):185-6.

[43] Knudson AG. Mutation and cancer: statistical study of retinoblastoma. Proceedings of the National Academy of Sciences 1971;68(4):820-3.

[44] Cairns J. Mutation selection and the natural history of cancer. Nature $1975 ; 255(5505): 197$.

[45] Nowell PC. The clonal evolution of tumor cell populations. Science $1976 ; 194(4260): 23-8$.

[46] Armitage P, Doll R. The age distribution of cancer and a multi-stage theory of carcinogenesis. British journal of cancer 1954;8(1):1.

[47] Stephens PJ, Greenman CD, Fu B, Yang F, Bignell GR, Mudie LJ, et al. Massive genomic rearrangement acquired in a single catastrophic event during cancer development. Cell 2011;144:27-40.

[48] Meyerson M, Pellman D. Cancer genomes evolve by pulverizing single chromosomes. Cell 2011;144(1):9-10.

[49] Shen M. Chromoplexy: A New Category of Complex Rearrangements in the Cancer Genome. Cancer Cell 2013;23(5):567-9.

[50] Cross WCH, Graham TA, Wright NA. New paradigms in clonal evolution: punctuated equilibrium in cancer. Journal of Pathology 2016;240(2):12636.

[51] Sato F, Saji S, Toi M. Genomic tumor evolution of breast cancer. Breast Cancer 2016;23(1):4-11.

[52] Notta F, Chan-Seng-Yue M, Lemire M, Li Y, Wilson GW, Connor AA, et al. A renewed model of pancreatic cancer evolution based on genomic rearrangement patterns. Nature 2016;538(7625):378-82. 
[53] Beckman RA, Schemmann GS, Yeang CH. Impact of genetic dynamics and single-cell heterogeneity on development of nonstandard personalized medicine strategies for cancer. Proceedings of the National Academy of Sciences 2012;109(36):14586-91.

[54] Melchor L, Brioli A, Wardell C, Murison A, Potter N, Kaiser M, et al. Single-cell genetic analysis reveals the composition of initiating clones and phylogenetic patterns of branching and parallel evolution in myeloma. Leukemia 2014;28(8):1705.

[55] McGranahan N, Swanton C. Biological and therapeutic impact of intratumor heterogeneity in cancer evolution. Cancer Cell 2015;12:15-26.

[56] Davis A, Gao R, Navin N. Tumor Evolution: Linear, Branching, Neutral or Punctuated? Biochimica et Biophysica Acta (BBA) - Reviews on Cancer 2017 ;

[57] Hodis E, Watson IR, Kryukov GV, Arold ST, Imielinski M, Theurillat JP, et al. A landscape of driver mutations in melanoma. Cell 2012;150:251-63.

[58] Burrell RA, McGranahan N, Bartek J, Swanton C. The causes and consequences of genetic heterogeneity in cancer evolution. Nature 2013;501:33845.

[59] Das Thakur M, Stuart DD. The evolution of melanoma resistance reveals therapeutic opportunities. Cancer Research 2013;73(20):6106-11.

[60] Shain AH, Yeh I, Kovalyshyn I, Sriharan A, Talevich E, Gagnon A, et al. The genetic evolution of melanoma from precursor lesions. The New England Journal of Medicine 2015;373(20):1926-36.

[61] Van Allen EM, Wagle N, Sucker A, Treacy DJ, Johannessen CM, Goetz EM, et al. The genetic landscape of clinical resistance to raf inhibition in metastatic melanoma. Cancer Discovery 2014;4:94-109. 
[62] Wagle N, Van Allen EM, Treacy DJ, Frederick DT, Cooper ZA, TaylorWeiner A, et al. Map kinase pathway alterations in braf-mutant melanoma patients with acquired resistance to combined raf/mek inhibition. Cancer Discovery 2014;4:61-8.

[68] Bosc C, Selak MA, Sarry JE. Resistance is futile: Targeting mitochondrial energetics and metabolism to overcome drug resistance in cancer treatment. Cell Metabolism 2017;26:705-7.

[69] Haq R, Shoag J, Andreu-Perez P, Yokoyama S, Edelman H, Rowe GC, et al. Oncogenic braf regulates oxidative metabolism via pgc1a and mitf. Cancer Cell 2013;23:302-15.

[70] Warburg O. On the origin of cancer cells. Science 1956;123(3191):309-14. 
[71] Hardeman KN, Peng C, Paudel BB, Meyer CT, Luong T, Tyson DR, et al. Dependence On Glycolysis Sensitizes BRAF-mutated Melanomas For Increased Response To Targeted BRAF Inhibition. Scientific Reports 2017;7(October 2016):42604.

[72] Delgado-Goni T, Miniotis MF, Wantuch S, Parkes HG, Marais R, Workman P, et al. Cancer Biology and Signal Transduction The BRAF Inhibitor Vemurafenib Activates Mitochondrial Metabolism and Inhibits Hyperpolarized Pyruvate-Lactate Exchange in BRAF-Mutant Human Melanoma Cells. Molecular Cancer Therapeutics 2016;15(12):2987-99.

[73] Través PG, de Atauri P, Marin S, Pimentel-Santillana M, Rodriguez-Prados JC, Marin de Mas I, et al. Relevance of the MEK/ERK Signaling Pathway in the Metabolism of Activated Macrophages: A Metabolomic Approach. The Journal of Immunology 2012;188(3):1402-10.

[74] Makinoshima H, Takita M, Saruwatari K, Umemura S, Obata Y, Ishii G, et al. Signaling through the phosphatidylinositol 3-kinase $(\mathrm{PI} 3 \mathrm{~K}) /$ mammalian target of rapamycin (mTOR) axis is responsible for aerobic glycolysis mediated by glucose transporter in epidermal growth factor receptor (EGFR)-mutated lung adenocarcinoma. Journal of Biological Chemistry 2015;290(28):17495-504.

[75] Hata RI, Izukuri K, Kato Y, Sasaki S, Mukaida N, Maehata Y, et al. Suppressed rate of carcinogenesis and decreases in tumour volume and lung metastasis in CXCL14/BRAK transgenic mice. Scientific Reports 2015;5:9083. 